\title{
Local Diversity and Fine-Scale Organization of Receptive Fields in Mouse Visual Cortex
}

\author{
Vincent Bonin, Mark H. Histed, Sergey Yurgenson, and R. Clay Reid \\ Department of Neurobiology, Harvard Medical School, Boston, Massachusetts 02115
}

\begin{abstract}
Many thousands of cortical neurons are activated by any single sensory stimulus, but the organization of these populations is poorly understood. For example, are neurons in mouse visual cortex — whose preferred orientations are arranged randomly—organized with respect to other response properties? Using high-speed in vivo two-photon calcium imaging, we characterized the receptive fields of up to 100 excitatory and inhibitory neurons in a $200 \mu \mathrm{m}$ imaged plane. Inhibitory neurons had nonlinearly summating, complex-like receptive fields and were weakly tuned for orientation. Excitatory neurons had linear, simple receptive fields that can be studied with noise stimuli and system identification methods. We developed a wavelet stimulus that evoked rich population responses and yielded the detailed spatial receptive fields of most excitatory neurons in a plane. Receptive fields and visual responses were locally highly diverse, with nearby neurons having largely dissimilar receptive fields and response time courses. Receptive-field diversity was consistent with a nearly random sampling of orientation, spatial phase, and retinotopic position. Retinotopic positions varied locally on average by approximately half the receptive-field size. Nonetheless, the retinotopic progression across the cortex could be demonstrated at the scale of $100 \mu \mathrm{m}$, with a magnification of $\sim 10 \mu \mathrm{m} /{ }^{\circ}$. Receptive-field and response similarity were in register, decreasing by $50 \%$ over a distance of $200 \mu \mathrm{m}$. Together, the results indicate considerable randomness in local populations of mouse visual cortical neurons, with retinotopy as the principal source of organization at the scale of hundreds of micrometers.
\end{abstract}

\section{Introduction}

Sensory stimuli are encoded through the receptive fields of large populations. In the visual cortex, even the smallest visual stimulus will activate the receptive fields of thousands of neurons (Van Essen et al., 1984; Bosking et al., 2002; Chen et al., 2006). The extent to which we understand this process depends on the size of the neural ensemble considered. The receptive fields of single neurons have been studied extensively (Hubel and Wiesel, 1959, 1962), showing selectivity for multiple attributes such as orientation, spatial phase, and retinotopic position.

The diversity of receptive fields within local populations is less well understood. Is the receptive field of a given neuron unique or do multiple neurons with similar receptive fields exist? In cat visual cortex, receptive fields of neighboring neurons measured from the same electrodes were rarely found to be similar (DeAngelis et al., 1999; Hetherington and Swindale, 1999). Diversity has

Received June 13, 2011; revised Oct. 7, 2011; accepted 0ct. 26, 2011.

Author contributions: V.B. and R.C.R. designed research; V.B. and M.H.H. performed research; V.B., M.H.H., and S.Y. contributed unpublished reagents/analytic tools; V.B. analyzed data; V.B., M.H.H., and R.C.R. wrote the paper.

This work was supported by NIH Grants R01 EY010115 and EY018742, and Microsoft Research. We thank Aaron Kerlin and Mark Andermann for help with the mouse preparation; Aaron Kerlin for the calibration data; Kenichi Ohki for helpful discussions; and Lindsey Glickfeld, Aaron Kerlin, Joshua Vogelstein, Mark Andermann, and John Maunsell for comments on the manuscript. We are greatly indebted to Joshua Vogelstein for discussions and for providing the code for non-negative deconvolution. We thank Adam Kampff for help with microscope design.

The authors declare no competing financial interests.

Correspondence should be addressed to either of the following: Vincent Bonin, Neuro-Electronics Research Flanders, VIB, Imec, K.U. Leuven, Imec-NERF, Kapeldreef 75, 3001 Leuven, Belgium. E-mail: vincent. bonin@nerf.be; or R. Clay Reid, Department of Neurobiology, Harvard Medical School, Goldenson 243, 220 Longwood Avenue, Boston, MA 02115. E-mail: clay_reid@hms.harvard.edu.

DOI:10.1523/JNEUROSCI.2974-11.2011

Copyright $\odot 2011$ the authors $\quad 0270-6474 / 11 / 3118506-16 \$ 15.00 / 0$ best been demonstrated using cellular imaging (Stosiek et al., 2003; Ohki et al., 2005) in local regions of the visual cortex of the mouse (Wang et al., 2006; Mrsic-Flogel et al., 2007; Sohya et al., 2007; Kerlin et al., 2010), where neurons with diverse properties are intermingled. Key to studying this diversity is the development of tools to measure the detailed receptive fields of comprehensive populations (Smith and Häusser, 2010).

One aspect of sensory representations that constrains diversity is organization. When neurons are organized over cortex according to their stimulus preferences, such as retinotopic position or stimulus orientation in carnivores and primates (Hubel and Wiesel, 1968, 1974; Tusa et al., 1978; Van Essen et al., 1984), the potential for diversity is more restricted. However, visual cortical organization is locally irregular in all species studied, with receptive fields of nearby neurons showing deviations from smooth mapping (Hubel and Wiesel, 1974; Van Essen et al., 1984; DeAngelis et al., 1999; Hetherington and Swindale, 1999), which increase diversity. Whether these deviations are organized at the scale of hundreds of micrometers is not known, particularly in rodents.

The mouse is emerging as an important model system for studying sensory processing and underlying neural circuitry. Its visual cortex is endowed with most major functional cell types identified in higher mammals (Dräger, 1975; Mangini and Pearlman, 1980; Niell and Stryker, 2008; Gao et al., 2010; Van den Bergh et al., 2010). Its retinotopic organization has been characterized at a coarse scale (Dräger, 1975; Wagor et al., 1980; Schuett et al., 2002; Kalatsky and Stryker, 2003). Its lack of orientation columns makes it possible to study functionally diverse local populations (Ohki et al., 2005; Wang et al., 2006; Sohya et al., 2007; Kerlin et al., 2010; Smith and Häusser, 2010). 
Here we present advances we made to study comprehensive receptive-field populations in the mouse visual cortex. We have combined high-speed cellular imaging with a rich random stimulus to characterize the spatial receptive fields of most neurons in a $200 \mu \mathrm{m}$ imaged plane. The results indicate considerable diversity in local populations with retinotopy as the principal source of organization at the scale of hundreds of micrometers.

Brief accounts of this study were published in abstract form (Bonin et al., 2009, 2010).

\section{Materials and Methods}

\section{Animal preparation}

All procedures were conducted in accordance with the ethical guidelines of the National Institutes of Health and were approved by the Institutional Animal Care and Use Committee at Harvard Medical School. Experiments were performed in GAD67-GFP $(\Delta$ neo $)$ mice in a C57BL/6 background (Tamamaki et al., 2003) of either sex, between 2 and 5 months of age. Anesthesia was induced and maintained with isoflurane (1-1.5\%) administered via a nose cone. A titanium head plate was attached to the skull (see Fig. 1) using dental cement, and a craniotomy was made ( $3 \mathrm{~mm}$ in diameter) over primary visual cortex (centered at 2.7-2.9 $\mathrm{mm}$ lateral from lambda, over the monocular region, as confirmed by receptive-field eccentricity; see Visual Stimulation, below). After dye injection, agarose (1.5\%; type III-A, Sigma-Aldrich) and a cover glass (World Precision Instruments) were applied to the surface of the brain to reduce brain motion. To maintain health and clarity, the contralateral eye was protected by a mouse contact lens (Sagdullaev et al., 2004) custom made from Aclar film (Ted Pella). The ipsilateral eye was sutured closed.

\section{Video-rate two-photon microscope}

Imaging was performed with a custom-designed two-photon laserscanning microscope (see Fig. $1 A$ ). Excitation light from a Mai Tai HP DeepSee laser (Spectra-Physics) with dispersion compensation was directed into a modulator (Conoptics) and a beam expander (Edmund Optics). The expanded beam was raster scanned onto the brain with a resonant (4 kHz, Electro-Optical Products) and a conventional galvanometer (Galvoline) (240 line frames, bidirectional, $31 \mathrm{~Hz}$ ) through a commercial scan lens (FV-PL-W3, Olympus), an achromatic tube lens (Thorlabs), and a 1.05 numerical aperture (NA) $25 \times$ objective lens (Olympus). To reduce fluctuations in scan amplitude due to heating of the resonant mirror by the laser beam, a shutter was placed between the scan and the tube lenses, and the scan mirrors were kept under constant illumination. Emitted photons were collected by a dichroic mirror (Chroma) located just above the objective lens and directed onto GaAsP photomultipliers (no cooling, $50 \mu \mathrm{A}$ protection circuit, $\mathrm{H} 7422 \mathrm{P}-$ 40MOD, Hamamatsu) through an infrared blocking filter (Chroma), a pair of aspheric lenses (Edmund Optics), and a set of blue (457 nm center; $50 \mathrm{~nm}$ band), green $(542 \mathrm{~nm} ; 50 \mathrm{~nm})$, and red $(629 \mathrm{~nm}, 53 \mathrm{~nm})$ filters (Semrock). The photomultiplier signals were amplified (DHPCA100 , Femto), and low-pass filtered (cutoff frequency $=\sim 700 \mathrm{kHz}$ ). These and the mirror driver signals were acquired at $3.3 \mathrm{MHz}$ using a multifunction data acquisition board (PCI-6115, National Instruments). Images were reconstructed in MATLAB and continuously streamed onto a RAID array. Microscope control was also performed in MATLAB using an analog output board (PCI-6711, National Instruments). The laser's dispersion compensation was adjusted to maximize collected fluorescence.

To separate the signals of multiple in vivo labels, we used different combinations of excitation wavelengths and emission filters (Sohya et al., 2007; Kerlin et al., 2010). Oregon Green Bapta-1 (OGB-1) and GFP were excited with 800 and $920 \mathrm{~nm}$. To image GFP after OGB loading, signals from the far blue tail of the GFP emission spectrum, where overlap with the OGB-1 emission spectrum is small, were averaged over a few minutes of imaging.

\section{Dye loading and two-photon imaging}

Under guidance of the two-photon microscope, a dye solution containing $1 \mathrm{~mm}$ Oregon Green 488 BAPTA-1 AM (Invitrogen) with 10\%
DMSO and Pluronic F-127 in ACSF (Stosiek et al., 2003) was injected near the center of the craniotomy, in a region free of blood vessels, $\sim 200-300 \mu \mathrm{m}$ below the cortical surface. We injected 20-40 short (1-2 s) pressure pulses through a glass pipette with an outer diameter of $\sim 2 \mu \mathrm{m}$. The injection solution also contained $60 \mu \mathrm{M}$ sulforhodamine-101 (Invitrogen) to label cortical astrocytes (Nimmerjahn et al., 2004). Data collection began 30-90 min after injection.

Planes of $200-300 \mu \mathrm{m}$ on a side were imaged $100-450 \mu \mathrm{m}$ below the pia mater. Each plane was continuously imaged for up to $2 \mathrm{~h}$ at constant average laser power of $10-60 \mathrm{~mW}$, measured at the objective front aperture. Individual experiment runs lasted 10-20 min. The field of view and imaging depth were readjusted between experiment runs to compensate for slow brain drifts.

\section{Visual stimulation}

Visual stimuli were generated in MATLAB and displayed using the software Presentation (Neurobehavioral Systems) on an LCD monitor (VX 922, Viewsonic), positioned $18 \mathrm{~cm}$ from the contralateral eye, spanning $130^{\circ}$ in azimuth and $105^{\circ}$ in elevation. The monitor was calibrated to an approximately linear luminance response curve by fitting the measured response curve with a gamma function (measured gamma $=2.19$ ) and applying the inverse gamma function to the stimulus intensities (mean luminance of $35 \mathrm{~cd} / \mathrm{m}^{2}$ ). The monitor refresh rate was $60 \mathrm{~Hz}$, and stimulus frames were updated at every second refresh $(30 \mathrm{~Hz})$. The resonant scanner and visual display each had their own clock. Synchronization was achieved off-line by measuring these clocks in the visual stimulation software (with a resolution of $0.1 \mathrm{~ms}$ ). Stimulus onset could show a temporal jitter of up to one video frame $(16.7 \mathrm{~ms})$ relative to the start of the scan frame. The monitor and the stimuli were placed at the location in visual field represented by the dye-loaded cortical volume, specifically in the visual periphery (azimuth $>60^{\circ}$ ), by monitoring the calcium responses to narrow stimuli of different azimuths and elevation. The retinotopic location of receptive fields depicted in Figures 4-8 was azimuth $70^{\circ}$ and elevation, $-10^{\circ}$.

For experiments using periodic stimuli (see Fig. 2), we stimulated with sinusoidal gratings moving in 8 or 12 different directions ( $100 \%$ contrast, temporal frequency of $1 \mathrm{~Hz}$, and spatial frequency of 0.02 cycles $\left./{ }^{\circ}\right)$ presented in sequential order followed by a blank stimulus (gray screen). Each stimulus was presented for $128 \mathrm{scan}$ frames $(\sim 4.2 \mathrm{~s})$ and was preceded by 128 scan frames $(\sim 4.2 \mathrm{~s})$ of uniform mean gray screen of mean luminance. Each stimulus was repeated three to five times.

For the receptive-field-mapping experiments, we constructed a stimulus made of randomly moving edges. The stimulus is based on the real oriented 3-D dual-tree wavelet transform (Selesnick et al., 2005). We chose this transform because its filters are oriented in space and in spacetime and therefore resemble visual cortical receptive fields. They were generated applying the inverse transform (using code by Shihua Cai and Keyong Li, Polytechnic Institute of New York University, New York, NY; http://eeweb.poly.edu/iselesni/WaveletSoftware/) to random sparse coefficients $(\sim 10 \%$ were nonzero). Coefficients had random signs, and their amplitudes were proportional to spatial scale to obtain a $1 / f$ spectrum. The stimulus included two to three spatial scales (range $0.01-0.1$ cycles $/^{\circ}$ ). The refresh rate was set to obtain a high-frequency cutoff of 2.5 $\mathrm{Hz}$. We generated $64 \times 64$ pixel stimulus frames covering a square $60^{\circ}$ on a side. To avoid correlations due to wrapping of the wavelet filters around the edges, we generated $128 \times 128$ pixel frames and cropped the center $64 \times 64$ pixels. We stimulated with epochs of wavelet stimuli lasting 512 scan frames $(\sim 16.5 \mathrm{~s})$. These were interleaved with epochs of uniform mean luminance lasting 256 scan frames $(\sim 8.25 \mathrm{~s})$ to allow on-line assessment of visual responsiveness and to limit the effects of adaptation (Niell and Stryker, 2008). Each trial included 16 stimuli for a total duration of $\sim 400 \mathrm{~s}$ and was repeated 3-15 times.

\section{Data analysis}

Data analysis was performed with MATLAB. To correct for motion along the imaged plane ( $x-y$ motion), the image stack was registered to the average field of view using efficient subpixel registration methods 
(Thévenaz et al., 1998; Guizar-Sicairos et al., 2008). Somatic regions of interest (ROIs) were identified using morphological filters (Ohki et al., $2005)$ and/or edited manually to correct mistakes. All visible somas were included without regard for sectioning ( $z$-position relative to imaged plane). The same ROI mask (set of ROIs) was used for all experiment runs at a given imaged plane. To compensate for slow brain drifts between runs, the ROI masks were shifted horizontally and vertically as estimated by coregistering the average images of each run.

To estimate spike-related somatic calcium activity, we calculated for each neuron a raw calcium time series, $F_{\text {raw }}$, summing pixel intensities over the neuron's ROI, and subtracting the estimated contributions of $z$-brain motion and neuropil contamination, as follows: $F_{\text {corrected }}=F_{\text {raw }}-F_{\text {motion }}-F_{\text {neuropil. }}$ We then expressed the time course as fractional signal change $R=\left(F_{\text {corrected }}-F_{0}\right) / F_{0} \times 100.0$, where $F_{0}$ is baseline fluorescence.

Computing $\mathrm{F}_{\text {motion }}$. We estimated $z$-motion by applying principal component analysis on a modified version of the registered stack. We cropped the registered stack to remove pixels on the edges, centered and normalized pixel values to zero mean and unit variance, and then scaled down pixel values inside the neuron ROIs by a factor of 100. A low-rank singular value decomposition (SVD) of this modified stack was performed using a fast, memory-efficient algorithm (Halko et al., 2011). We selected the first $k$, components dominated by motion and scanning artifacts (range $0-16$ ), based on visual inspection of their spatial and temporal profiles. From the $k$ components, we computed a low-rank approximation of the normalized stack, denormalized the result, and summed the pixels over the neurons' ROIs. The resulting signals captured rapid motion due to heartbeat and breathing, as well as slow brain drifts and other slow variations such as bleaching (Fig. $2 A-D$ ). In experiments in which motion was small and responses were large, sensory responses were visible in the first principal components and the correction $F_{\text {motion }}$ was not used ( $k$ was set to 0 ).

Computing $\mathrm{F}_{\text {neuropil }}$. We estimated the time course and magnitude of neuropil contamination as follows. We estimated neuropil time courses by summing a ring of pixels around the somas and by applying a lowrank SVD. The time courses' weights, $w$, were chosen to maximize the skewness of $F_{\text {corrected }}$. The values of $w$ ranged between $0.4-1.4$. For the few cells for which the optimization did not converge or gave aberrant results ( $w \leq 0$ or $w>2.0$ ), we used the median $w$ of the remaining neurons. Similar results were obtained by maximizing kurtosis instead of skewness.

Computing $\mathrm{F}_{0}$. We devised a robust method to estimate baseline fluorescence $F_{0}$ from the measured time course $F_{\text {corrected }}$. The mean $(\bar{F})$, at any time scale, reflects both baseline and spike-related activity, and so is not appropriate to estimate baseline alone. We determined the baseline as the smallest constant $k$ such that the cumulative sum $C(t)=$ $\sum_{t^{\prime}=0}^{t^{\prime}}\left(F_{\text {corrected }}\left(t^{\prime}\right)-k\right)$ is monotonically increasing at long timescales (8 $s)$. When $k$ is correct, $C(t)$ is constant during periods of no spike activity. Thus, this method detects when silent periods occur and uses them to determine the baseline. Specifically, we calculated $C(t)$ with $k=\bar{F}$, decimated it by 256 samples, and smoothed it with a Savitzky-Golay filter (order 4, frame size 21). We then fitted the result with piecewise linear functions to compute its derivative $\Delta C(t)$. Finally, we estimated $F_{0}$ by calculating the 10th percentile of $\Delta C(t)$.

Inferring firing activity. To estimate firing activity, we applied the model-based deconvolution method of Vogelstein et al. (2010) onto the time courses $R$ using code provided by the authors. The model consists of a Poisson spike generator, an exponential filter of amplitude $A$ and time constant $\tau$, and a Gaussian noise source of amplitude $\sigma$. All model parameters were held fixed. The filter time constant $\tau$ was set to $1 \mathrm{~s}$, and the noise amplitude $\sigma$ to one to two times the SD of the time courses $R$ in the absence of a visual stimulus. The method outputs a time series of real positive numbers, $\hat{n}$, that is proportional to firing rates, and we calculated $A \hat{n} / T$, where $T$ is the sample interval ( $\sim 31 \mathrm{~ms})$ to express the results as transient rates in percentage $\Delta F / F \mathrm{~s}^{-1}$. To compare fits with the observed calcium time courses, we convolved $\hat{n}$ with the exponential filter.

Responses to gratings. We averaged the inferred firing across repetitions of the stimulus and calculated for each stimulus epoch the mean response (known as $\mathrm{F} 0$ in the literature, not to be confused with $F$ for fluorescence above and previously) and the peak magnitude of the harmonic response (F1) at the temporal frequency of the grating. We quantified the selectivity of responses by computing the circular variance of mean responses (Ringach et al., 2002). We quantified the periodicity of responses as the ratio of the F1 component over the F0 component. We calculated the preferred orientation by expressing responses as vectors in the polar plane in which magnitudes correspond to response magnitude and angles are set to twice the stimulus orientation, and then calculating half the angle of the vector sum (Niell and Stryker, 2008).

Reverse correlation analysis. To estimate receptive fields from the responses to the wavelet stimulus, we first computed event-triggered averages. We summed the stimulus frames that preceded calcium events by $0-7$ scan frames, weighting the frames by the magnitudes of the calcium events. Because spike-related calcium signals typically change fluorescence by several percent, we excluded events with magnitudes smaller than $1 \% \Delta F / F$ (30 ms bins). We calculated a separate triggered average for each of the stimulus epochs, which resulted in 16 event-triggered averages for each repetition of the stimulus. We then performed a jackknife analysis, resampling the collection of event-triggered averages 20 times, each time omitting a distinct $5 \%$ of the data. We calculated the sample mean and SE and then expressed the result as the ratio of the mean over the SE ( $z$-scores).

Signal correlations. We computed the trial average of inferred firing activity in response to the wavelet stimuli, summed this into $500 \mathrm{~ms}$ bins (16 samples), and calculated the Pearson correlation coefficient between responses. We defined signal correlation as the correlation at zero time delay less the correlation lagged by 1 bin. The latter corrects for effects of ongoing activity, which may not have been averaged out. We only considered neurons for which model fits predicted $>10 \%$ of variance in the stimulus-locked responses. Epochs of gray screen were also excluded for this analysis.

\section{Results}

We wished to study the local diversity and organization of receptive fields in mouse primary visual cortex. Two-photon calcium imaging is well suited for studying neural populations (Stosiek et al., 2003; Kerr et al., 2005), but its application for measuring receptive-field properties has generally been limited to one or two stimulus attributes (Ohki et al., 2005; Mrsic-Flogel et al., 2007; Sohya et al., 2007; Kerlin et al., 2010; Ko et al., 2011).

Here we present advances we have made to study receptive fields of comprehensive visual cortical populations. We first present methods to image in vivo the time-resolved activity of approximately 100 cortical neurons simultaneously (Figs. 1, 2). Using these methods, we characterized the receptive fields of excitatory and inhibitory neurons with simple sine-wave grating stimuli, studying their tuning and linearity of spatial summation (Fig. 3). We then measured the spatial receptive fields of excitatory neurons using a novel random stimulus and reverse correlation (Fig. 4) and extracted their parameters using a simple model (Fig. 5). We used model fits to study the local diversity and organization of receptive fields (Fig. 6), their retinotopic mapping across the cortical surface (Fig. 7), and their relation to stimulus-evoked correlated activity (Fig. 8). Together, the results indicate considerable diversity in local populations of mouse visual cortical neurons, with retinotopy as the principal source of organization at the scale of hundreds of micrometers.

\section{Wide-field video-rate imaging of neural populations}

To study the activity of populations of neurons in the cortex of mice, we built a video-rate two-photon microscope for calcium imaging (Fig. 1). The microscope, based on a resonant scanner (Fig. 1A), could image $>300 \mu \mathrm{m}$ fields of view (Fig. $1 B$ ) at a rate 

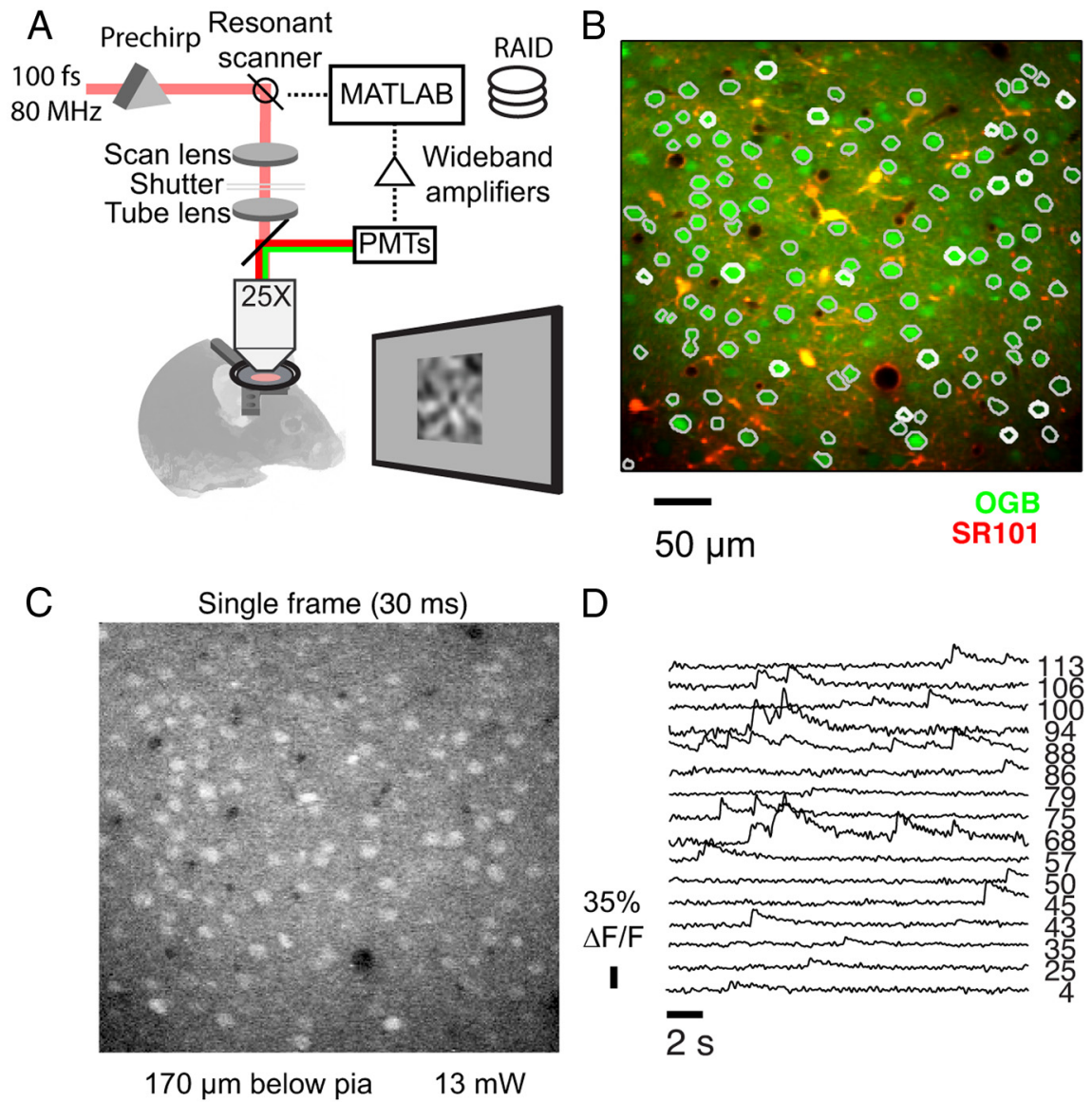

Figure 1. Wide-field, video-rate, two-photon imaging in mouse visual cortex. A, In vivo imaging preparation. Mice were anesthetized and their heads held fixed while visual stimuli were displayed to the contralateral eye. For imaging, short infrared laser pulses (100 fs) were raster scanned (frame rate $31 \mathrm{~Hz}$ ) onto the brain using a resonant galvanometric system ( 8000 lines/s, 240 lines per frame) through a high-NA water-immersion objective lens. Emitted light was collected with GaAsP photomultipliers (PMTs) and acquired using a multifunction board ( $0.3 \mu \mathrm{s} / \mathrm{sample})$. Images were reconstructed on-line in MATLAB and streamed onto a disk array (RAID). B, Typical imaged plane showing neurons (green) and astrocytes (yellow) labeled with calcium indicator OGB-1 and sulforhodamine 101 (SR101). Overlaid contours (gray and white) indicate manually segmented neuron cell bodies. C, Single-image frame ( $g r e e n$ channel) showing that cell bodies and blood vessels are visible at $30 \mathrm{~ms}$ time scale ( $170 \mu \mathrm{m}$ below pia, $13 \mathrm{~mW}$ average power). $\boldsymbol{D}$, Somatic calcium time courses obtained by summing pixels over cell bodies, for 16 of the 114 labeled neurons ( $\boldsymbol{B}$, thick contours). At these low laser powers, a slow decay of fluorescence and transient amplitude was observed (see Results).

of 31 images per second (see Materials and Methods). The microscope yielded high signal-to-noise images using relatively low average laser power (Fig. 1C) $(\sim 10-20 \mathrm{~mW}$ at $150-250 \mu \mathrm{m}$ depth) and allowed the measurement of somatic calcium fluorescence of $>100$ neurons simultaneously (Fig. 1D). At these low laser power levels, we could image for up to $90 \mathrm{~min}$ with limited changes in absolute fluorescence or in the magnitude of evoked responses $\left(1-F_{\min } / F_{\max }<15 \%, \Delta F / F_{0} \sim 0.9-1.3 \% \mathrm{~s}^{-1}\right.$, eight planes).

We devised a simple procedure to correct for the effects of brain motion and neuropil contamination on the somatic calcium time courses (Fig. 2) (see Materials and Methods). Small cell movements are common during in vivo imaging due to heartbeat, respiration, and, in awake animals, voluntary movements (Göbel and Helmchen, 2007). These movements cause cell bodies to move in and out of the regions of interest, causing fluorescence changes that can be as large as those caused by neural activity (Fig. $2 B 1$, first row). To correct for brain motion, we aligned each image frame (Fig. 2A), and applied principal component analysis (Mitra and Pesaran, 1999; Mukamel et al., 2009) to the somatic regions to estimate the components due to $z$-motion (Fig. 2 B1, second row) as well as to neuropil regions to obtain a measure of contamination (Fig. 2B1, third row). We then computed somatic time courses by averaging fluorescence over cell bodies and subtracting the estimated motion and neuropil components (Fig. 2 B1, fourth row, black). We used these corrected time courses to estimate firing activity (Fig. 2B1, fourth row, red).

This procedure effectively corrected for the effects of brain motion and neuropil contamination (Fig. $2 B-E$ ). The correction worked for poorly sectioned cells (Fig. 2B1, left column), which could be strongly affected by motion, and for well sectioned cells (Fig. 2 B1, right column), which were generally less affected. The procedure removed the common signals that are shared between time courses (Fig. $2 C$ ), thereby allowing a more precise characterization of coactive ensembles (Fig. $2 E)$. Because images were acquired at video rate, we could remove non-spikerelated signals at various time scales (Fig. $2 \mathrm{D1})$, including breathing $(<2 \mathrm{~Hz})$ and heart beat $(9 \mathrm{~Hz})$. The corrected time courses have a near perfect exponential amplitude spectrum (Fig. 2D2, thick black curve) as expected from the time course of spike-related calcium fluorescence (Smetters et al., 1999; Kerr et al., 2005; Kerlin et al., 2010). Importantly, the correction procedures made no assumption about the frequency spectrum of the components.

To reconstruct firing rates from the calcium signals, we used the model-based deconvolution method of Vogelstein et al. (2010) (see Materials and Methods). We selected this method because it is fast and allows reconstruction of firing rates while the experiment is taking place. Based on a generative model of calcium responses, the method outputs a time series of positive real numbers that are proportional to the neurons' firing rates (Fig. 2 B1, red ticks). We expressed these deconvolved time courses as calcium transient rate (percentage $\Delta F / F^{-1}$ ). The method explained the time course of responses (Fig. 2 B1, red curves), capturing their amplitude spectrum (Fig. 2D2, red curve), as evidenced by the flat spectrum of the residuals (Fig. 2D2, gray curve). When tested on simultaneous calcium imaging and cell-attached recordings in a similar in vivo preparation (Kerlin et al., 2010), the firing rate estimates were highly consistent with the measured spiking (Fig. 2 B2). The correlation coefficient between the measured and estimated firing rates was $0.81 \pm 0.05(n=3$ cells $)$ for regular spiking neurons, and $0.37 \pm 0.15(n=5)$ for fast-spiking neurons (eight frames or $240 \mathrm{~ms}$ bins). The lower performance of the method for fast-spiking neurons was largely attributable to the small field of view in these calibration experiments, typically a single cell, which made it difficult to compensate for slow baseline 
A

$X Y$ motion

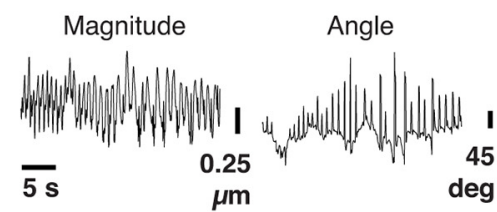

B1

Uncorrected

Poorly sectioned

Well sectioned
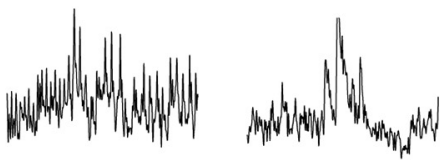

Z-motion

component
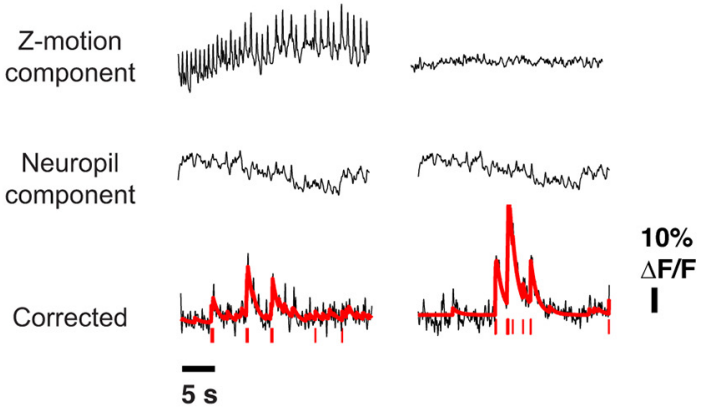

\section{B2}

Calcium transient rate

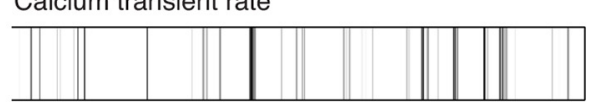

Cell attached

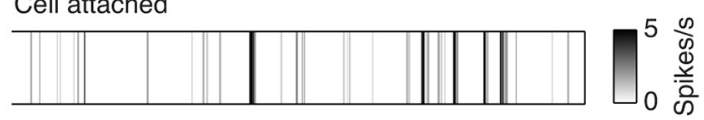

$\overline{50 s}$
C

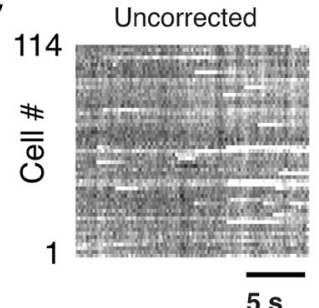

D

1

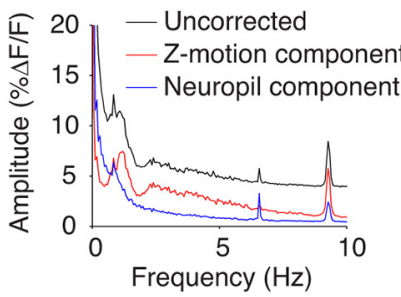

2

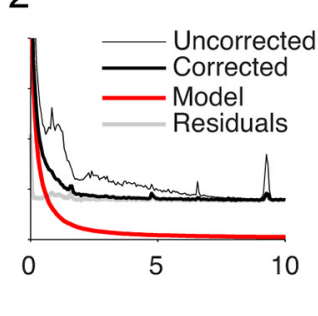

E

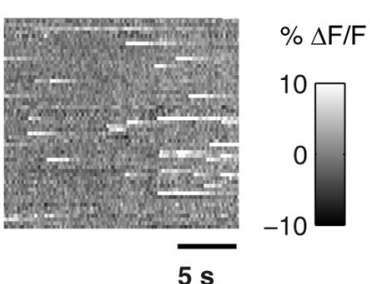

12
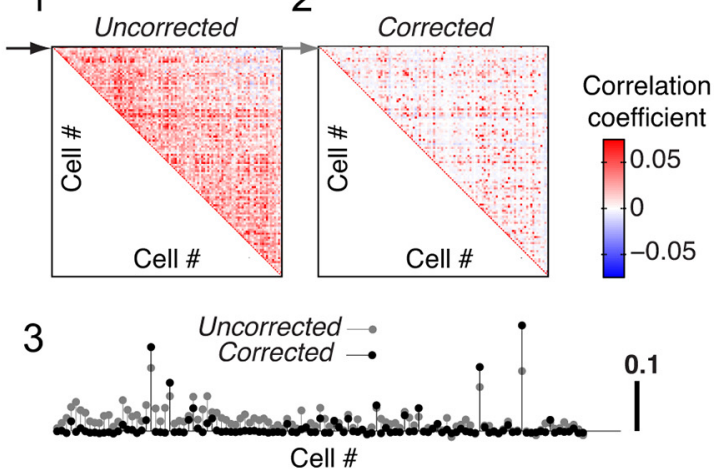

Figure 2. Estimating spike-related calcium activity in the face of motion and neuropil contamination. $A$, Frame-to-frame $x-y$ brain displacement in polar coordinates estimated from rigid registration of the image stack to the average frame. $\boldsymbol{B 1}$, Correction for motion in depth (z-motion) and neuropil contamination. Somatic fluorescence time courses of two nearby cells obtained from registered stack (first row), estimated z-motion component (second row), estimated neuropil component (third row), and corrected time courses (fourth row, black). Note how the correction captures the effect of $z$-motion on the cells' time courses. Model somatic calcium fluorescence (fourth row, red curves) and firing events (fourth row, red ticks, $\Delta F / F>3.0 \%, 30 \mathrm{~ms}$ bins) inferred using a fast non-negative deconvolution algorithm (Vogelstein et al., 2010). B2, Validation of the method of Vogelstein et al. (2010) on simultaneous calcium imaging and cell-attached recordings in mouse visual cortex in vivo. Raster plots ( $500 \mathrm{~ms}$ bins) show calcium transient rates (top) and measured firing (bottom) rates of a regular-spiking (putative excitatory) neuron. Data from a previous study (Kerlin et al., 2010). C, Uncorrected versus corrected time courses. Half the imaged cells are shown for clarity. The correction removes the common, periodic signals that contaminate the raw somatic time courses. $\boldsymbol{D}$, Amplitude spectra of uncorrected time courses (top, black), estimated z-motion components (D1, red), and estimated neuropil components (D1, blue). In the $z$-motion component, effects of breathing $(<2 \mathrm{~Hz})$ and heart beat $(9 \mathrm{~Hz})$ are visible. In the neuropil component, rhythmic calcium activity $(<2 \mathrm{~Hz})$ and ambient light $(7 \mathrm{~Hz})$. The corrected time courses ( $\mathbf{D 2}$, thick line) have an exponential spectrum, which was well described by the deconvolution method (D2, red), as inferred by the flat spectrum of the residuals [corrected—-model] (D2, gray). $\boldsymbol{E}$, Pairwise correlation between estimated firing of simultaneously imaged neurons before (E1) and after correction (E2). Stem plot (E3) compares a single row of the correlation matrices (E1, E2, arrow). Correcting for motion and neuropil reveals the sparse correlation structure of responses. C, E, Data from Figure 1.A, B, D, Data from Figures $4-8$.

drifts. This problem generally did not arise when imaging large fields (Fig. 2C).

\section{Spatial summation in excitatory and inhibitory neurons}

We first studied the spatial summation properties of excitatory and inhibitory neurons. The two major functional cell types in visual cortex are simple and complex cells, which differ in the spatial properties of their receptive fields as originally described in the cat (Hubel and Wiesel, 1962). Simple cells have receptive fields with segregated ON and OFF subregions that are elongated along the axis of the preferred orientation. Complex cells are also tuned for orientation but have receptive fields with spatially overlapping ON and OFF response fields (ON/OFF receptive fields). Spike recordings in mouse visual cortex also identified a third physiological type, with ON/OFF receptive fields and broad tuning for orientation (Dräger, 1975; Niell and Stryker, 2008). Recent electrophysiological and imaging studies in transgenic animals suggest that these nonselective complex-like cells may correspond to GABAergic interneurons (Sohya et al., 2007; Liu et al., 2009; Kerlin et al., 2010).

To determine whether excitatory and GABAergic neurons indeed show distinct modes of spatial summation, we studied the timing of their calcium responses. In the cat, simple and complex cells can be distinguished from the timing of their firing responses to sine-wave grating stimuli (Movshon et al., 1978a,b). These stimuli elicit periodic responses in simple cells but not in complex cells, which show more tonic responses. We studied calcium responses in visual cortex of GAD67-GFP knock-in mice whose GABAergic interneurons are labeled with GFP (Tamamaki et al., 2003). We stimulated with sinusoidal gratings moving at $1 \mathrm{~Hz}$ in different directions and used the method of Vogelstein et al. (2010) to infer neural firing. We then compared the tuning and the periodicity of responses between GFP-positive and GFPnegative neurons. Our ability to resolve the time course of cal- 

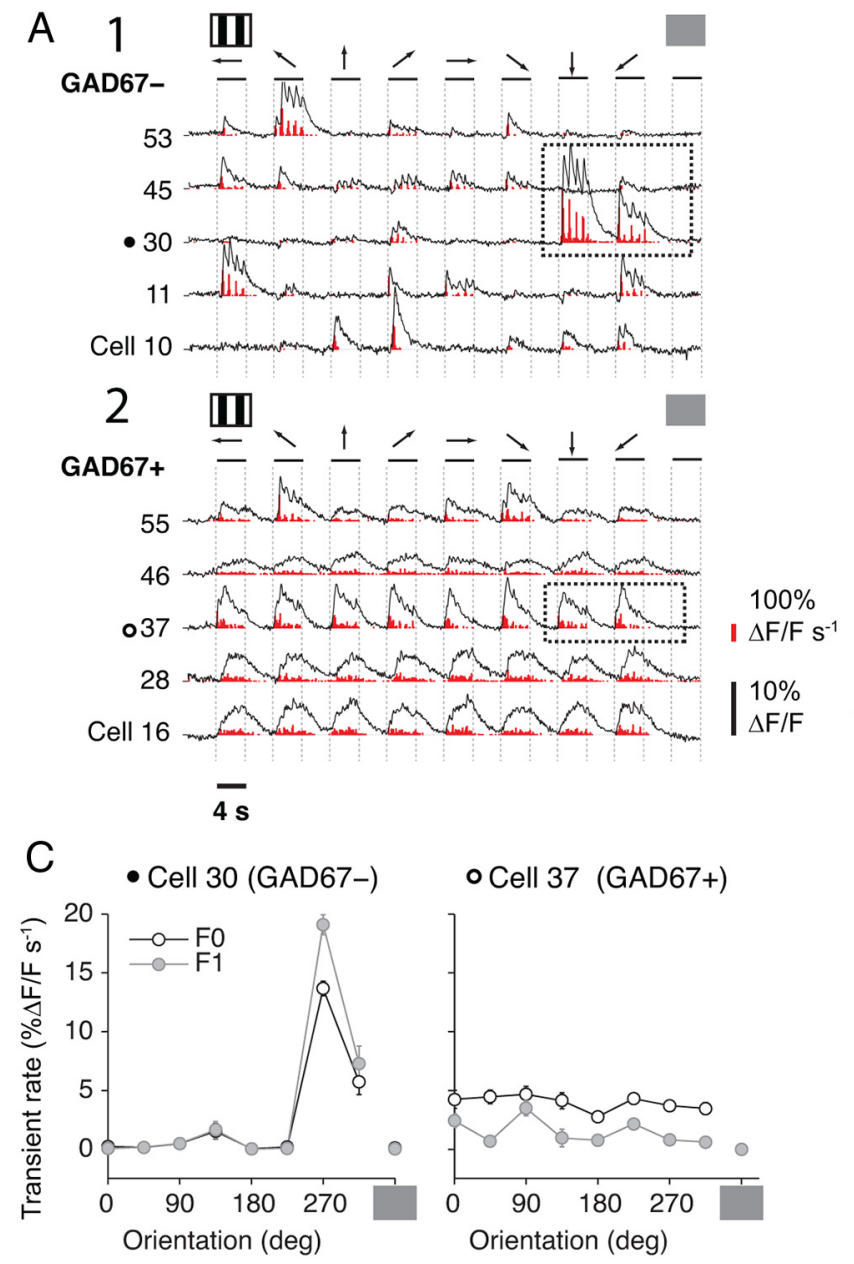
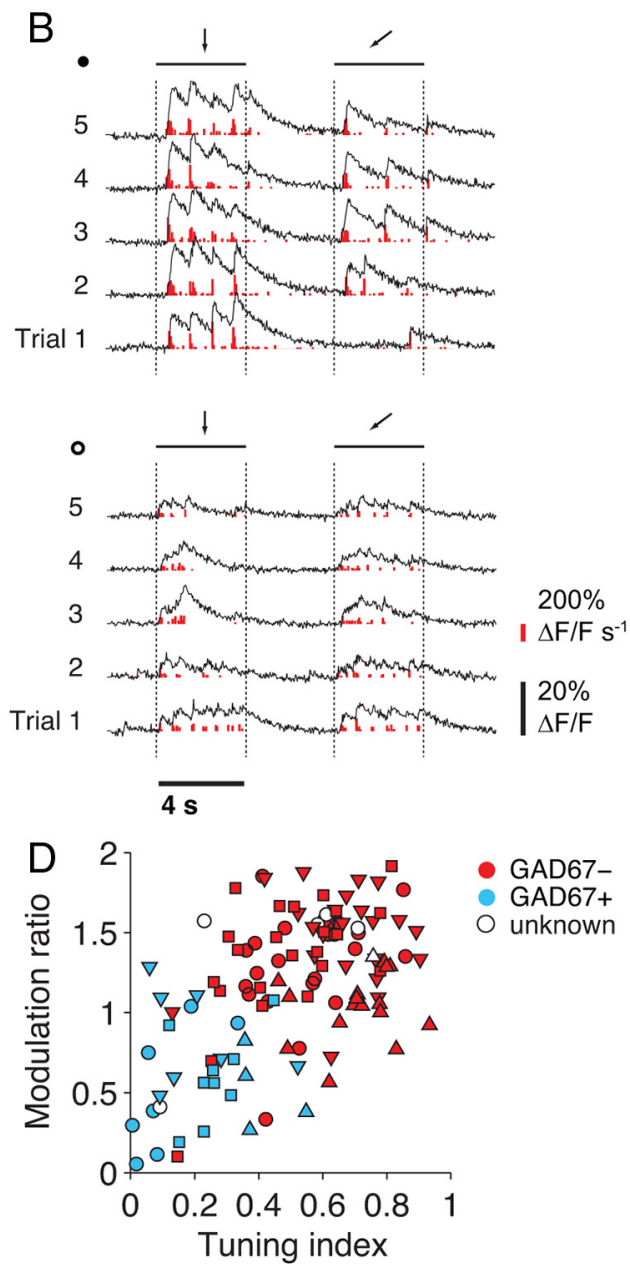

Figure 3. Spatial summation in excitatory and inhibitory neurons. Experiments in visual cortex of GAD67-GFP knock-in mice in which all GABAergic inhibitory interneurons are labeled with green fluorescent protein (Tamamaki et al., 2003). Stimuli were sinusoidal gratings moving at $1 \mathrm{~Hz}$ in $8-12$ different directions interleaved with periods of isoluminant gray screen. $A$, Trial average somatic calcium responses (black traces) and transient rates (red histograms) for five excitatory (A1) and five inhibitory neurons (A2) (averaged across five repetitions). Vertical lines indicate stimulus onset and offset. Excitatory neurons were highly selective for a narrow range of stimulus orientations and often showed pronounced periodic responses to these stimuli. GABAergic interneurons were nonselective or weakly selective and exhibited little periodicity in their responses. $\boldsymbol{B}$, Single-trial responses of an excitatory neuron (closed circle) and an inhibitory interneuron (open circle, same data as in $\boldsymbol{A}$, dashed boxes). $\boldsymbol{C}$, For the same neurons, tonic (open symbols) and periodic (close symbols) components of responses as a function of stimulus direction. Error bars indicate $S E$. $\boldsymbol{D}$, Modulation versus tuning indices for 262 neurons ( $N=4$ planes from two animals). Data points for each imaged plane depicted with different symbols. Excitatory neurons (red) and interneurons (blue) show distinct functional properties.

cium responses at a resolution of tens of milliseconds (Fig. 1), free of the artifacts that tend to be concentrated at low temporal frequencies (Fig. 2), made these experiments possible.

Neurons responded strongly to the grating stimuli (Fig. 3). Over a third of the imaged cells showed large stimulus-driven calcium transients $(\Delta F / F>10 \%, N=103 / 262$ cells from two planes in two animals). Of these cells, $\sim 15 \%$ were GAD67 positive. Consistent with past studies (Sohya et al., 2007; Niell and Stryker, 2008; Kerlin et al., 2010; Zariwala et al., 2011), responses of excitatory neurons were highly selective for a narrow range of stimulus orientations (Fig. 3A1), while responses of inhibitory neurons were nonselective or weakly selective (Fig. 3A2) (but see Ma et al., 2010; Runyan et al., 2010). We quantified response selectivity with a tuning index whose value ranges from 0 , for not tuned, to 1, for highly tuned (see Materials and Methods). The tuning index for inhibitory responses was significantly lower than for excitatory responses $(0.22 \pm 0.05$ vs $0.59 \pm 0.02$, mean $\pm \mathrm{SE})$.

Responses of excitatory and inhibitory neurons differed strikingly in their time courses. Because somatic time courses were sampled at video rate $(31 \mathrm{~Hz})$, we could resolve periodic modu- lations of responses at a range of frequencies. We found that excitatory neurons often showed a strong periodic response at the temporal frequency of the grating (Fig. 3A1), indicative of simple receptive fields with segregated $\mathrm{ON}$ and OFF subregions (Movshon et al., 1978a; Niell and Stryker, 2008). This modulation was visible in both the calcium time courses (Fig. $3 A 1$, black traces) and the inferred firing activity (Fig. 3A1, red histograms). GABAergic interneurons, in contrast, showed an increase in fluorescence with little periodic response (Fig. 3A2), suggestive of complex-like receptive fields with ON/OFF responses (Movshon et al., 1978b; Niell and Stryker, 2008).

These functional differences in orientation tuning and response modulation were highly correlated with cell types. To quantify the periodicity of responses, we calculated the harmonic component of the inferred firing at the temporal frequency of the stimulus (Fig. 3C, gray) and compared it to the average response (Fig. 3C, black). These response components closely followed each other in excitatory neurons (Fig. 3C, left) but less so in GABAergic neurons (Fig. $3 C$, right). The modulation ratio-the ratio of the periodic response to the mean response-is signifi- 


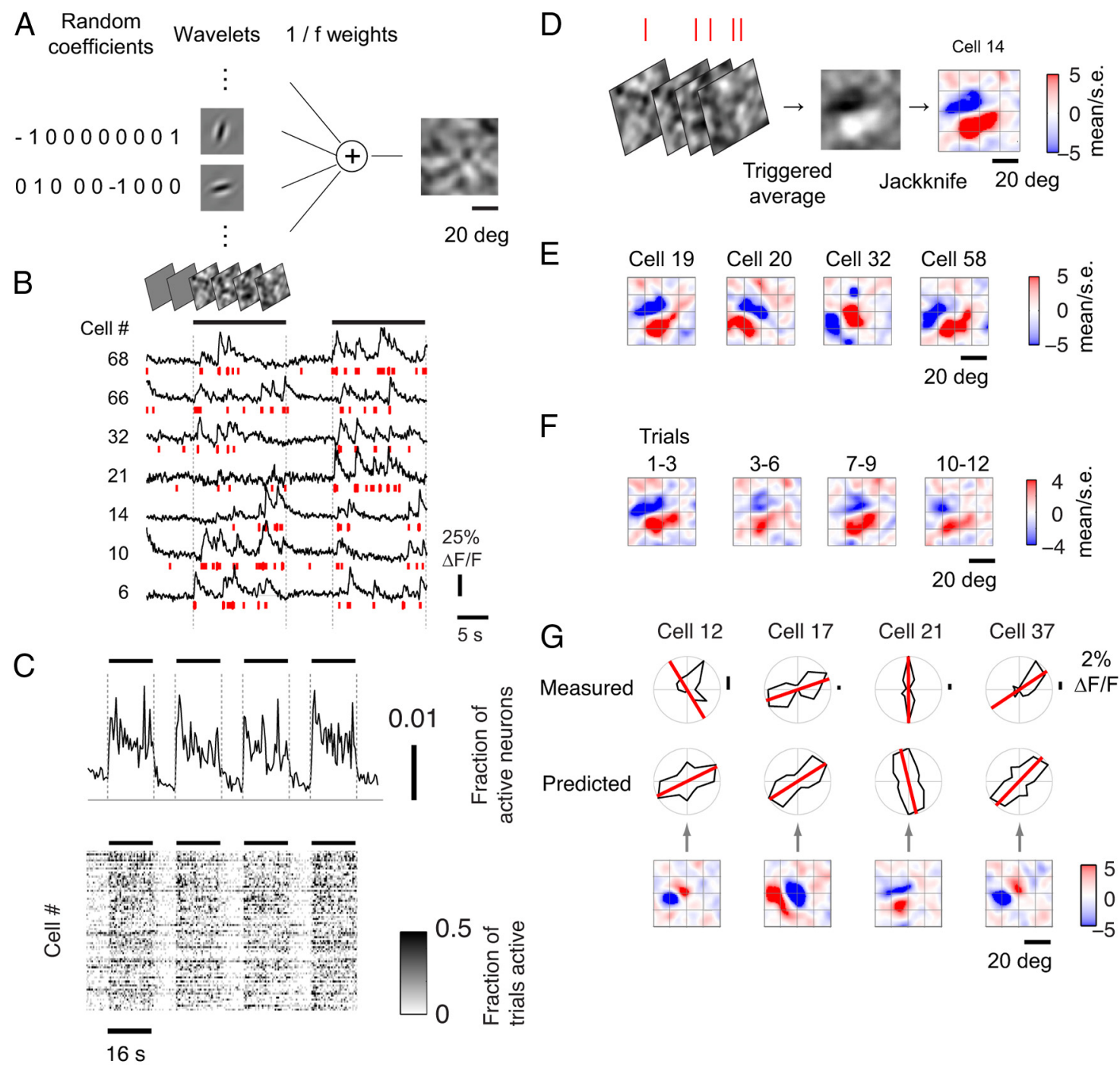

Figure 4. Characterizing excitatory neurons with wavelet stimuli. $A$, Wavelet stimulus made of random, localized, oriented stimuli of different positions, sizes, and directions. The stimulus is generated by applying the inverse 3D wavelet transform to sparse random coefficients. B, Single-trial calcium time courses (black) and inferred firing activity (red) for 7 of 82 imaged cells (one imaged plane). Stimulus consists of $\sim 16 \mathrm{~s}$ epochs of wavelets preceded by $\sim 8 \mathrm{~s}$ periods of gray screen ( 16 different stimuli for a total of $\sim 400 \mathrm{~s}$ per trial). Same experiment as in Figure $2, A$, $B 1$, and $D$, and Figures $5-8$. C, Same experiment, average fraction of neurons active (top) and fraction of trials active as function of time (bottom) $(\Delta F / F>3.0 \%, 500 \mathrm{~ms}$ bins). $D$, Triggered averages computed by calculating the cross-correlation between the stimulus and the response $(\Delta F / F>3.0 \%)$, and by comparing results between stimulus repetitions. $E$, Receptive fields often have elongated ON (red) and OFF (blue) subregions characteristic of simple cells. $\boldsymbol{F}$, Receptive fields estimated from successive sets of trials are similar (20 min of imaging each; cell 14). $\mathbf{G}$, Comparison to responses to moving grating stimuli. Orientation tuning (black lines) and preference (red lines) inferred from receptive-field mapping is consistent with that measured with drifting gratings.

cantly lower in interneurons than in excitatory neurons (Fig. 3D) $(0.60 \pm 0.09$ vs $1.32 \pm 0.04$, mean $\pm \mathrm{SE} ; N=15$ and 84 cells $)$. The depth of modulation was highly correlated with the selectivity of responses for orientation $(r=0.60, p<1 \mathrm{e}-10, t$ test). Excitatory and inhibitory neurons were readily distinguished by these properties (Fig. 3D, red and blue symbols). These differences were robust. Stimulus-locked transients were visible in single trials in excitatory neurons (Fig. 3B, top) but not in interneurons (Fig. 3B, bottom).

While excitatory neurons and interneurons differ in the size and decay constant of their spike-related calcium fluorescence (Kerlin et al., 2010), these functional differences are not likely to reflect differences in the calcium-buffering properties of the neurons. At the stimulus frequency $(1 \mathrm{~Hz})$, our ability to resolve firing rate modulations is not limited by the decay time in fluorescence, but its rise time, which is similar in both cell types (Kerlin et al., 2010, less than one imaging frame or $63 \mathrm{~ms}$ ). We confirmed the validity of this assertion using simulations (data not shown).
Thus, excitatory neurons in visual cortex often have simple receptive fields, and GABAergic neurons have receptive fields that are complex-like but, unlike classical complex cells seen in species such as cats and primates, are broadly tuned for stimulus orientation (Dräger, 1975; Niell and Stryker, 2008; Liu et al., 2009; Kerlin et al., 2010; Liu et al., 2010).

\section{Characterizing receptive fields with wavelet stimuli}

We next devised a method to measure the spatial receptive fields of excitatory neurons. Receptive fields have been effectively studied with random stimuli (Jones and Palmer, 1987a; Reid and Shapley, 1992; Reid and Alonso, 1995; Reid et al., 1997; DeAngelis et al., 1999; Hetherington and Swindale, 1999). However, stimulation with white, uncorrelated stimuli such as random single spots (sparse stimuli) or multiple spots (dense stimuli) produces small responses (Ringach et al., 1997; Liu et al., 2009; Ma et al., 2010) and can drive only a fraction of the neurons simultaneously. To obtain a dense sampling of receptive fields with calcium 
A

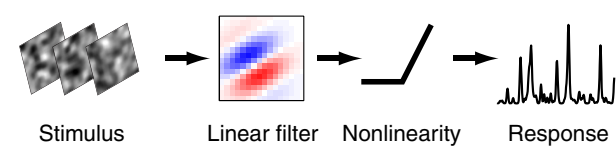

B

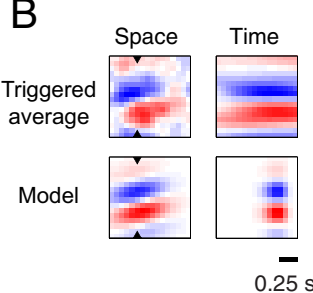

C

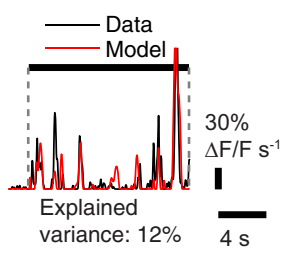

D

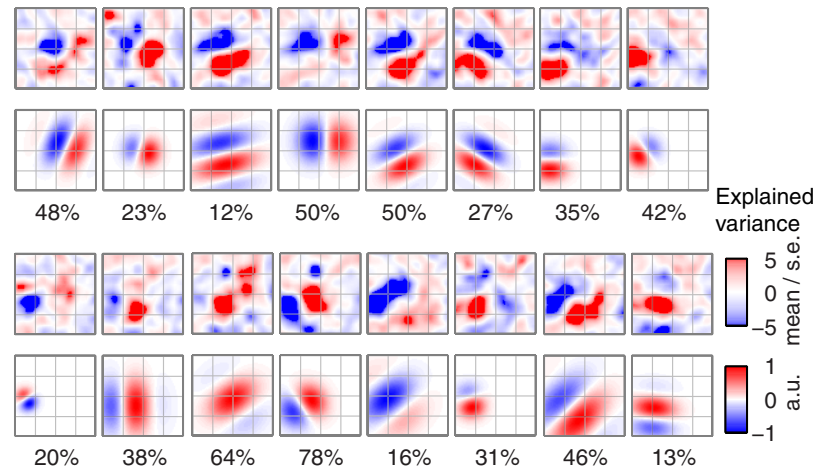

Figure 5. Receptive-field modeling. $\boldsymbol{A}$, Model of responses consisting of a linear filter (a Gabor filter in space and a gamma function in time) and a rectifying nonlinearity. $\boldsymbol{B}$, Fitted linear filter for neuron in Figure $4 D$ (cell 14, note change in color scale in Fig. 4D). C, Measured (black line) and model responses (red line). For this cell, the model fit explains $\sim 12 \%$ of the stimulus-evoked variance in the responses. Responses were smoothed with a Gaussian filter $(\sigma=30 \mathrm{~ms})$. $\boldsymbol{D}$, Example estimated spatial receptive fields. Event-triggered averages (top rows, as computed in Fig. 4) and estimated spatial linear filters (bottom rows). Only fits that explain $>10 \%$ of the stimulus-evoked variance are shown. From these fits, we can extract parameters such as receptive-field position, size, and orientation.
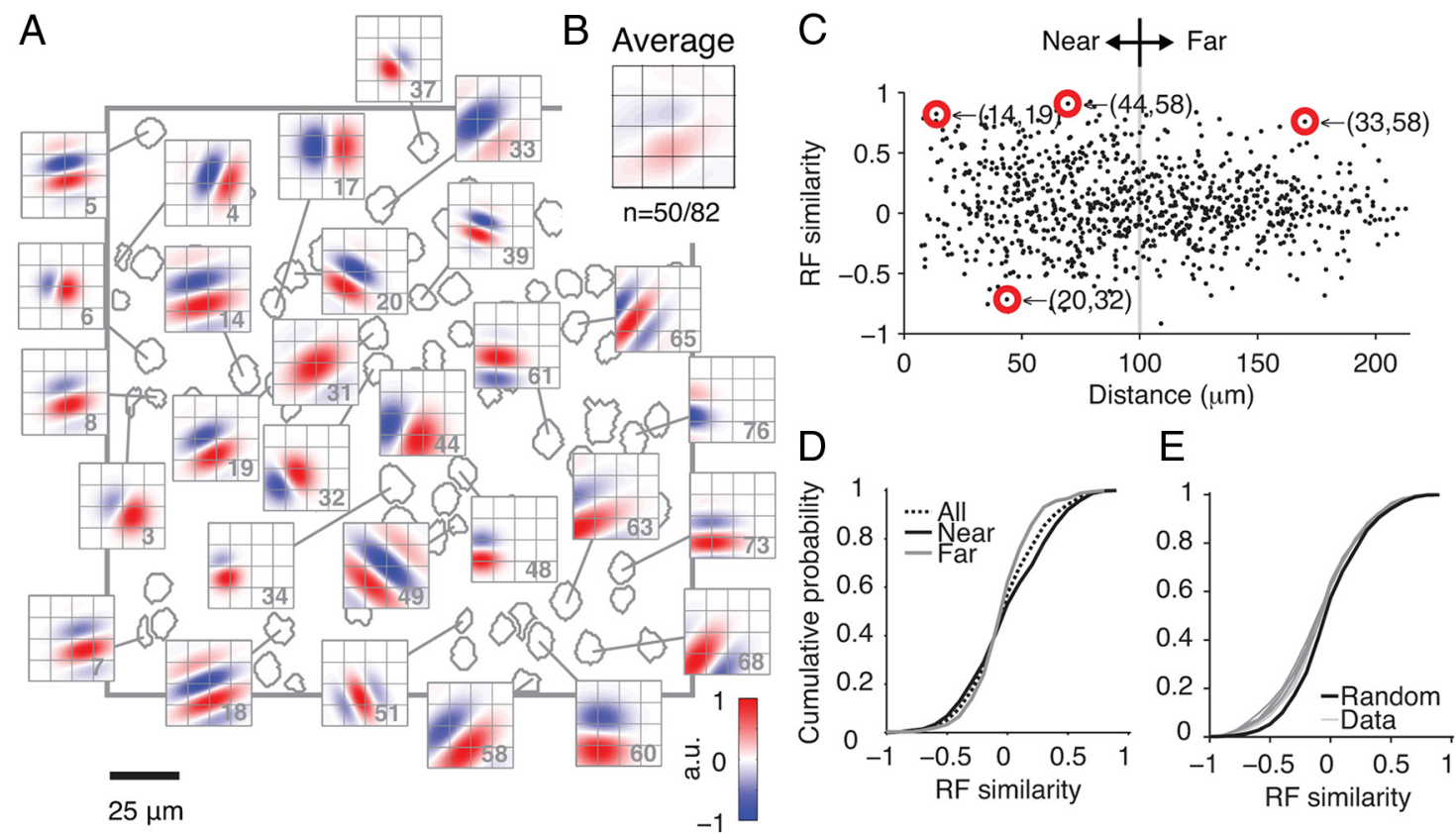

Figure 6. Local diversity and organization of spatial receptive fields. $\boldsymbol{A}$, Model spatial receptive fields (RFs) overlaid on a schematic view of cortex (gray contours) to illustrate relationship between RF position and neuron location in cortex. Lines point to the cell bodies of neurons from which the RFs were recorded. Only a subset of the most significant RFs are shown (absolute mean/SE $>5.0 \%$ and explained variance $>5 \%, n=50 / 82$ cells). Receptive fields with different properties are intermingled. Note the shift in receptive-field position with neuron location (left to right, see Fig. 7). B, Average RF in imaged plane. ON and OFF RF subregions were biased toward distinct regions of visual space, but segregation was not strict, as evidenced by the diversity seen in $\boldsymbol{A}$ and by the small magnitude of the average (peak $\sim 0.25$ ). $\boldsymbol{C}$, Receptive-field similarity versus distance between cell bodies. Similarity defined as the normalized dot product between RFs. D. Cumulative distributions of pairwise RF similarity for neurons $<100 \mu \mathrm{m}$ part (black), for neurons $100-250 \mu \mathrm{m}$ apart (gray), and for all neurons combined (dashed). $\boldsymbol{E}$, Cumulative distributions of RF similarity for measured (black) and surrogate RFs (gray). Surrogate RFs obtained by assigning random orientations or phases to the measured RFs. Results for multiple randomized sets are overlaid. Only significant RFs are considered.

imaging, we needed a dense, structured stimulus that produces robust firing responses (Niell and Stryker, 2008).

We designed a wavelet stimulus to produce responses that are easily detectable using calcium imaging. The stimulus consists of localized oriented stimuli, or wavelets (Selesnick et al., 2005), whose positions, sizes, and directions were chosen randomly (Fig. 4A). The wavelets resemble Gabor functions, which have been used in the past to model the receptive fields of visual cortical simple cells (Jones and Palmer, 1987b; Ringach et al., 2002).
We chose their sizes to match the properties of mouse visual cortical neurons (Niell and Stryker, 2008) and their density so that only a few wavelets were on the display at any given time (see Materials and Methods).

The stimulus evoked strong calcium responses from the neurons (Fig. $4 B$ ). We stimulated with periods of wavelets interleaved with epochs of gray screen. Responses consisted of large calcium fluorescence transients followed by a slow decay to baseline (Fig. $4 B$, traces) from which we estimated the timing and the 
A

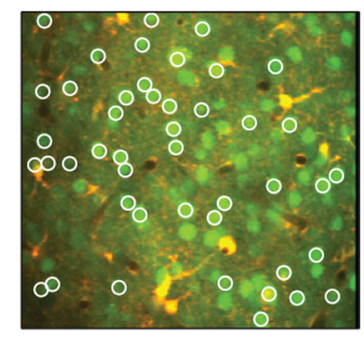

$25 \mu \mathrm{m}$

C
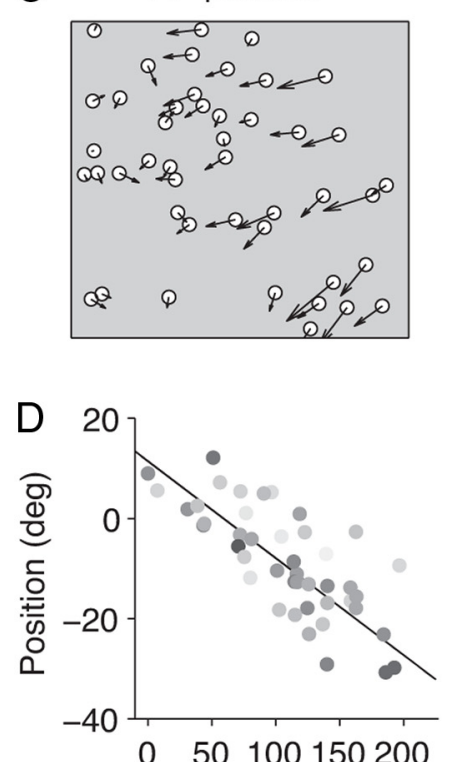

Distance along $v_{1}(\mu \mathrm{m})$
B

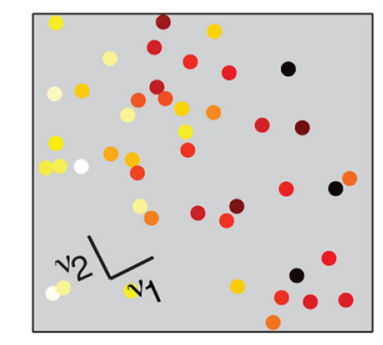

\section{RF Elevation}

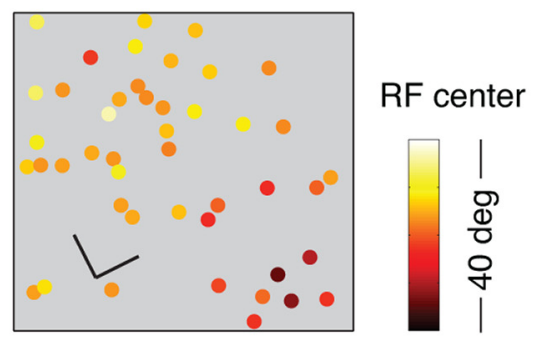

\section{Smooth map}
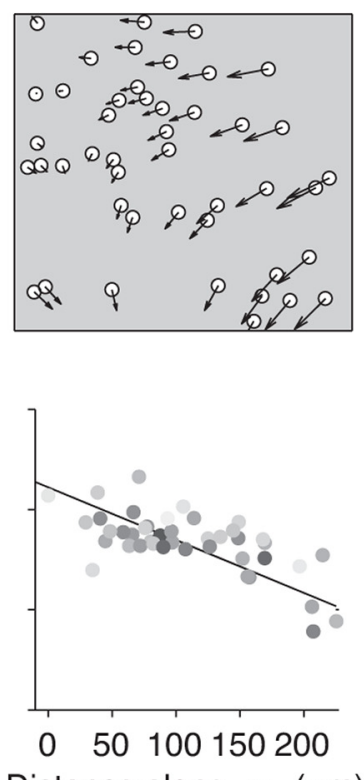
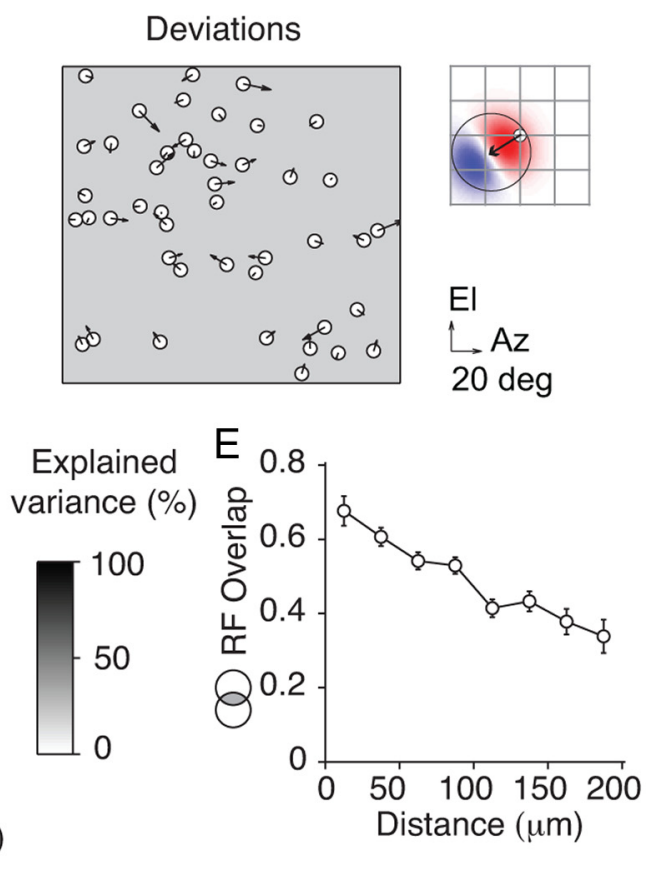

Figure 7. Local retinotopic organization. $A$, Field of view with characterized neurons (circles). Only fits that explain $>5 \%$ of the stimulus-evoked variance are considered ( $N=50 / 82$ neurons). $\boldsymbol{B}$, Cell body positions (circles) and receptive-field centers relative to stimulus center (color-coded) along azimuth (left) and elevation (right). Vectors $\nu_{1}$ and $\nu_{2}$ denote the perpendicular axes along which RF azimuth and elevation show the largest shifts. C, Receptive-field positions (left), estimated retinotopic map (middle), and positional scatter (right). Maps show cell body (circles) and receptive-field centers (arrows; see inset on right). Retinotopic map defined as the rigid transformation of cortical space that minimized total square error of positions. $D$, Projection of retinotopic map (lines) and positions (circles) along vectors $\nu_{1}$ (left) and $\nu_{2}$ (right). For this dataset, cortical magnification is $5.1 \mu \mathrm{m} /{ }^{\circ}$ along $\nu_{1}$ and $10.1 \mu \mathrm{m} /{ }^{\circ}$ along $\nu_{2}$. E, Receptive-field overlap versus distance between cell bodies. Overlap defined as the ratio of shared receptive-field area over the area of the smaller receptive field. Area calculated from the width of the Gaussian envelope (full-width at half-maximum). Error bars indicate SE.

magnitude of firing events (Fig. $4 B$, red). We calculated the average percentage of calcium activity (percentage $\Delta F / F$ ) for the periods of gray and for the periods of wavelet stimuli. Many neurons showed increased calcium activity upon stimulation ( 290 of 536 cells, $N=7$ planes in two animals, $z$-score $>3.0$, range $30-85 \%$ of identified somas of each plane), with an average deconvolved fluorescence signal of $0.75 \pm 0.06 \% \Delta F / F \mathrm{~s}^{-1}$ during the epochs of gray screen and $1.32 \pm 0.18 \% \Delta F / F \mathrm{~s}^{-1}$ upon stimulation (mean \pm SE, 290 cells, $N=7$ planes). The calcium transient frequency $(\Delta F / F>3 \%, 30 \mathrm{~ms}$ bins $)$ and magnitudes increased upon stimulation $(0.03 \pm 0.01 \mathrm{~Hz}$ and $0.76 \pm 0.06 \%$ $\Delta F / F$ during gray screen, and $0.11 \pm 0.02 \mathrm{~Hz}$ and $1.32 \pm 0.18 \%$ during stimulation, mean $\pm \mathrm{SE}, 290$ cells, $N=7$ planes). The peak transient magnitude also increased upon stimulation $(5.20 \pm 0.52 \%$ to $7.74 \pm 0.98 \%$, 99th percentile, mean $\pm \mathrm{SE})$. Responses were very sparse with only a small fraction of all neurons simultaneously active (Fig. $4 C$, top). Yet the stimulus was very effective, yielding time-locked responses in most cells (Fig. $4 C$, bottom).
From these responses, we calculated event-triggered averages as initial, approximate measures of the receptive fields (Fig. 4D). We computed the cross-correlation between the stimulus and the response, and compared results across repetitions to express them as $z$-scores (mean/SE). The triggered averages had segregated and elongated ON (Fig. 4D, red) and OFF subregions (Fig. 4D, blue), characteristic of visual cortical simple cells (Fig. $4 E$ ). Receptive fields were obtained in as little as 20 min of imaging (three repetitions of the stimulus) (Fig. $4 F$ ), comparable to the time required for measuring receptive fields with electrode recordings of responses to structured stimuli (Niell and Stryker, 2008).

The measured receptive fields were consistent with the tuning of responses to oriented stimuli. In some experiments, we also measured responses to moving sinusoidal gratings and compared these responses to the predictions obtained from the estimated receptive fields (Fig. 4G). There was agreement between the measured and predicted orientation tuning curves (Fig. 4G, black and red lines). The mean ( $\pm \mathrm{SE}$ ) difference in preferred orientation was $4.1 \pm 5.1^{\circ}(n=61)$, and the mean absolute difference was 

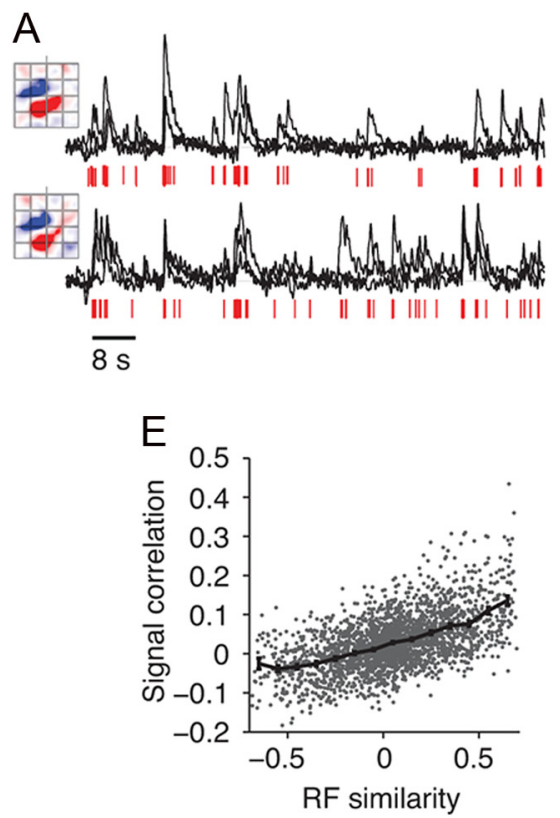

B

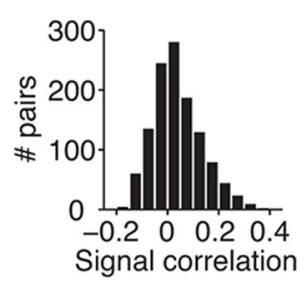

$\mathrm{F}$

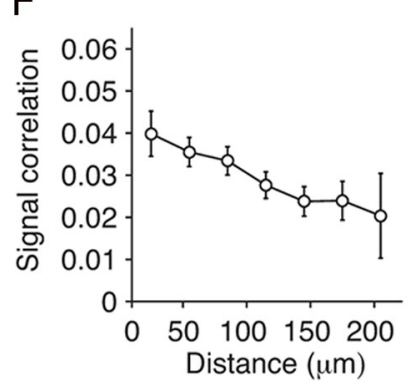

C

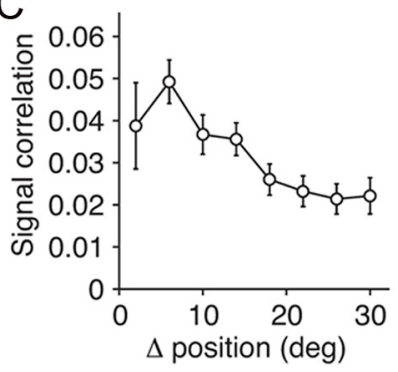

$\mathrm{G}$

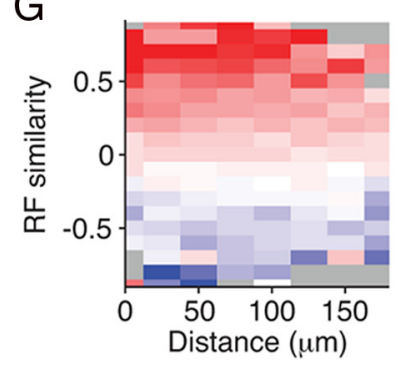

D

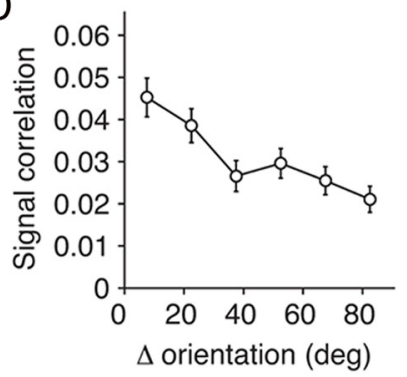

Figure 8. Receptive fields and activity correlations. $\boldsymbol{A}$, Signal trial calcium responses (black) of two excitatory neurons with similar spatial receptive fields (insets) and inferred firing (red, $>3 \%$ $\Delta F / F)$ for three repetitions of the wavelet stimulus. $\boldsymbol{B}$, Distribution of signal correlations. Signal correlation defined as correlation coefficient between trial-average responses of pairs of neurons at zero lag minus the correlation lagged by 1 bin ( 500 ms bins). The calculated signal correlation for the cell pair shown in $\boldsymbol{A}$ is $0.35 \pm 0.08$ (mean \pm SD bootstrap). $\boldsymbol{C}-\boldsymbol{F}$, Average signal correlation (black lines and symbols) versus absolute distance in receptive-field position $(\boldsymbol{C})$, absolute difference in receptive-field orientation $(\boldsymbol{D})$, receptive-field similarity $(\boldsymbol{E})$, and distance between cell bodies $(\boldsymbol{F})$. Gray dots in $\boldsymbol{E}$ show individual neuron pairs. Error bars indicate SE. G, Histogram shows average signal correlation as function of cortical distance and receptive-field similarity. For a given degree of similarity, correlation is approximately constant.

$31.5 \pm 3.1^{\circ}(n=61)$. The predicted tuning was generally broader than the measured responses, likely because the analysis did not include a spiking mechanism, which acts to sharpen the tuning of responses (Priebe and Ferster, 2008). The triggered averages of dozens of neurons could be measured simultaneously. Overall, the experiments yielded triggered averages for approximately half the imaged neurons (peak $z$-score $>5.0, N=263$ of 533 imaged cells, $N=7$ planes in 2 animals), and for up to $80 \%$ of cells in some imaged planes.

Thus, high-speed calcium imaging of responses to random stimuli can reveal the receptive fields of dense populations. Critical to these experiments was our ability to estimate firing rates free of motion artifacts and the use of a stimulus that evoked large responses from many neurons (Fig. $4 B, C$ ).

\section{Receptive-field model and parameters}

To study receptive-field diversity and organization, we needed to quantify receptive-field parameters. The event-triggered averages are blurred by the spatial structure and the slow time course of the stimulus, and therefore cannot be used to estimate receptive-field parameters without bias (Theunissen et al., 2001). Receptive fields can be quantified by fitting a model to the responses (Carandini et al., 1997). This provides receptive-field parameters and quantifies the degree to which the receptive fields explain the neural responses.

We fitted a linear rectification model to explain responses and to extract receptive-field parameters. The model consists of a linear filter whose output is a time-varying signal, analogous to an intracellular potential, followed by a rectifying nonlinearity to obtain firing rates (Carandini et al., 1997). The linear filter consisted of a spatial receptive field, which filters the visual stimulus through a Gaussian window-oriented sinusoidal function (a Gabor function), (Jones and Palmer, 1987b; Ringach, 2002; Niell and Stryker, 2008), and a temporal filter (a gamma function) (Cai et al., 1997), which delays and smooths the responses (Fig. 5A). We fitted the model to the trial average of deconvolved responses smoothed with a Gaussian filter $(\sigma=30 \mathrm{~ms})$. Using fits of the event-triggered averages as initial parameters, we estimated the model parameters that minimized the squared error between measured and model responses.

The model fits provided a simple description of the neurons' receptive fields. The estimated spatial filters captured the main features of the event-triggered averages (Fig. $5 B, D$ ), providing a method to measure receptive-field parameters. The estimated temporal filters showed less blur than the corresponding triggered averages (Fig. 5B), indicating that the least-square fits account for the correlations contained in the stimulus. As a result, the model output is more temporally precise and can explain some of the calcium responses locked to the stimulus (Fig. 5C). To assess the quality of fits, we calculated the percentage of stimulus-locked variance in the responses that is explained by the model output. Model performance varied across neurons, explaining up to $75 \%$ of the variance in the stimulus-locked responses of the neurons (Fig. 5D). We considered fits that had predictive power (explained variance $>10 \%, N=222$ of 463 imaged cell bodies, six planes in two animals). The average size of the spatial filters was $\sim 12^{\circ}\left(12.1 \pm 6.3^{\circ}\right.$, mean $\pm \mathrm{SD}$, half-width at half-maximum), and the average delay of the temporal filters was generally $<250 \mathrm{~ms}(140 \pm 78 \mathrm{~ms}$, median \pm interquartile range; $N=181$ ). These results are commensurate with similar measurements using electrode recordings (Niell and Stryker, 2008; Gao et al., 2010).

Local receptive-field populations: diversity and organization We used model fits to study the receptive fields' diversity and organization. Are spatial receptive fields unique within their surrounding populations or do they show redundancy? How is this diversity distributed over the visual space and over the 
imaged plane? Receptive fields of nearby neurons could be arranged randomly or could show clustering, with nearby cells having similar receptive fields. By addressing these questions, we constrain how local populations of excitatory neurons sample the visual scene.

Receptive fields of different orientations and positions were scattered over the imaged field (Fig. 6A). Nearby neurons could have similar receptive fields (Fig. $6 \mathrm{~A}$, cells 14 and 19), receptive fields with overlapping position but different orientations (Fig. 6A, cells 58 and 60), or receptive fields with different positions and orientations (Fig. $6 A$, cells 17 and 20). In addition, distant neurons could have similar receptive fields (Fig. 6A, cells 33 and 58). This random arrangement of receptive-field orientation and position is consistent with the arrangement of orientation preference measured with grating stimuli (Ohki et al., 2005; Wang et al., 2006; Sohya et al., 2007; Kerlin et al., 2010), and with the scatter of ON and OFF receptive-field subregions of nearby neurons measured with sparse noise (Smith and Häusser, 2010).

The receptive fields, however, were not entirely random. First, as reported by Smith and Häusser (2010), ON and OFF subregions fell on average onto spatially offset regions of the visual field (Fig. $6 \mathrm{~B}$ ). This segregation was not strict; overlapping subregions of opposite signs were also frequently observed (Fig. $6 A$ ), and, as a result, the overall bias was small compared with the local diversity (Fig. $6 B$, note the small magnitude of the average receptive field, peak $\sim 0.25)$. Second, despite local scatter, a global retinotopic shift in receptive-field position was often discernible across the cortical surface (Fig. 6A, left to right). These measurements, to our knowledge, are the first to unambiguously demonstrate the mapping of receptive field position in a small patch of cortex. We study retinotopy further in the next section (Fig. 7).

To quantify receptive-field diversity, we calculated for each neuron pair in the imaged plane an index of similarity and studied its distribution. We defined similarity as the dot product between the normalized spatial filters (DeAngelis et al., 1999; Usrey et al., 1999; Alonso et al., 2001), which mirrors the spatial filtering of the visual scene performed by the neurons. This measure equals 1 for identical receptive fields, 0 for dissimilar receptive fields (orthogonal or nonoverlapping), and -1 for receptive fields of opposite signs. We considered for each plane the neurons for which we obtained significant event-triggered averages (mean/SE >5.0) and for which the model explains $>5 \%$ stimulus-evoked variance $(50 / 82$ cells for the plane in Fig. 6 and 192/455 cells, six planes in two animals).

According to this measure, nearby neurons generally had quite dissimilar spatial receptive fields (Fig. $6 \mathrm{C}$ ). The similarity distribution showed a peak at zero and few counts near 1 and -1 where highly similar and oppositely signed receptive fields should fall (Fig. 6D). This degree of diversity is more pronounced than in the cat visual cortex where highly similar receptive fields were more frequently observed (DeAngelis et al., 1999), probably reflecting the difference in functional organization between the visual cortices of the two species. In cat visual cortex, nearby simple cells have similar orientation preference (Hubel and Wiesel, 1959), so spatial receptive fields can only differ in their position or phase.

We then investigated whether there was any clustering across the cortex of functionally similar neurons. We related receptivefield similarity to the distance between cell bodies, as inferred from the hand segmentation mask (Fig. 6A). If there were clustering over short cortical distances, then similarity should depend on cortical distance. Overall, there was only a weak dependence of similarity on cortical distance (Fig. 6C). Neurons $<100 \mu \mathrm{m}$ apart had more similar receptive fields (Fig. $6 D$, solid line) than neurons between 100 and $200 \mu \mathrm{m}$ apart (Fig. $6 D$, gray line), reflecting a slow fall off in receptive-field overlap with cortical distance (Fig. 7E). Thus, aside from retinotopy, receptive fields are disorganized over cortex.

We next studied the clustering of receptive fields in visual space. Are receptive fields more similar than would be expected from random sampling of visual features? To test this hypothesis, we recalculated the similarity indices assigning receptive fields random orientations or phases while maintaining their positions. The similarity distribution in these surrogate data differed slightly from the measured (Fig. $6 E$ ). As expected from the clustering of $\mathrm{ON}$ and OFF subregions, randomization increased the frequency of oppositely signed receptive fields (Fig. 6E, left of ordinate). However, randomization only mildly affected the frequency of highly similar receptive fields (Fig. $6 E$, right of ordinate), suggesting that the receptive fields show about the same level of clustering as expected from random sampling.

\section{Local receptive-field populations: retinotopy and scatter}

Thus far, the principal source of organization we observed was retinotopy, the map of receptive-field position across the cortical surface. Retinotopy has been studied with electrode recordings (Hubel and Wiesel, 1974; Dräger, 1975; Van Essen et al., 1984) and optical imaging (Grinvald et al., 1994; Schuett et al., 2002; Kalatsky and Stryker, 2003). Electrode recordings of nearby neurons in primate (Hubel and Wiesel, 1974; Dow et al., 1981), cat (Das and Gilbert, 1997; DeAngelis et al., 1999; Hetherington and Swindale, 1999), and mouse (Dräger, 1975; Wagor et al., 1980) have shown deviations from smooth retinotopy. This scatter may reflect true deviations or it may reflect the sampling of cell bodies (and other cell processes) at a distance of the electrode tip (Hetherington and Swindale, 1999). Our imaging data can resolve this question.

We estimated global retinotopy from the model fits (Fig. 7). When we plotted receptive-field centers against the position of cell bodies (Fig. 7A), a progression of receptive-field azimuth and elevation could be seen along two orthogonal directions across the cortical surface (Fig. 7B). This retinotopic map was well described by a simple 2D rigid transformation of cortical space (Fig. 7C). From this transformation, we could estimate cortical magnification, the distance in cortex that corresponds to an average $1^{\circ}$ displacement of receptive-field centers. The magnification factor along azimuth and elevation was $8.2 \pm 2.3 \mu \mathrm{m} /{ }^{\circ}$ (mean $\pm \mathrm{SD}$, $N=6$ planes in 2 animals). This is slightly lower than measurements made at the millimeter scale at a range of eccentricities (Dräger, 1975; Wagor et al., 1980; Schuett et al., 2002; Kalatsky and Stryker, 2003) $\left(\right.$ range $\left.12-24 \mu \mathrm{m} /{ }^{\circ}\right)$.

We then estimated receptive-field scatter (Fig. 7D). The receptive-field centers (Fig. $7 D$, symbols) showed clear deviations from the estimated smooth retinotopy (Fig. $7 D$, lines). This scatter likely does not reflect uncertainty in receptivefield measurements; the magnitude of deviations and the degree to which receptive fields capture the responses were unrelated (Fig. $7 D$, gray shades). Similar results were obtained by considering ON and OFF subregions separately (data not shown). We quantified scatter as the SD of receptive-field center position minus the estimated global retinotopy. Across experiments, scatter was $7.0 \pm 2.2^{\circ}$ (mean $\pm \mathrm{SD}, N=6$ planes), amounting to about one-half the size of a typical receptive field $\left(\sim 12^{\circ}\right.$, see previous section). 
Thus, cellular imaging can resolve the global progression of receptive-field position across cortex as well as deviations in nearby neurons. The deviations likely reflect true receptive-field scatter, not measurement noise. The receptive-field scatter we observed in mouse V1 is approximately one-half the size of a typical receptive field.

\section{Local receptive-field populations: relation to correlated activity}

Receptive fields only provide a simplified account of visual responses, so the estimated receptive fields might miss important response properties. To investigate properties not reflected in the receptive fields, we studied signal correlations in the responses to our wavelet stimuli (see Materials and Methods). Signal correlations measure the degree of similarity in evoked responses between pairs of neurons (Di Lorenzo, 1989; Gawne and Richmond, 1993) and, unlike receptive fields, account for both the linear and nonlinear aspects of the responses. In the primate visual cortex, signal correlations are generally low (Vinje and Gallant, 2000; Reich et al., 2001; Yen et al., 2007), indicating a low degree of redundancy in the responses. Signal correlations have been shown to predict functional connections between the neurons (Ko et al., 2011). Yet little is known about how signal correlations are organized in local populations.

We investigated the magnitude of correlations and related them to model fits. We calculated the Pearson correlation coefficient between the binned trial-averaged responses (500 ms bins) and defined signal correlation as the correlation at zero time delay minus the correlation lagged by 1 bin (to correct for correlations due to ongoing activity). We only considered neurons for which model fits predicted $>10 \%$ of variance in the stimulus-locked responses and excluded nonstimulus epochs (gray screen) from the analysis.

Responses were only weakly correlated (Fig. 8). Neurons with similar receptive fields showed correlated responses but often responded at distinct time points of the stimulus (Fig. $8 \mathrm{~A}$ ). Consistent with the weak correlations observed in the responses to natural scenes (Ko et al., 2011), some neurons showed high correlations, but most did not (Fig. $8 \mathrm{~B}$ ). Signal correlations were strongest for cells with similar receptive-field positions (Fig. 8C) and orientations (Fig. $8 D$ ), confirming that the spatial receptive fields make a significant contribution to signal correlation. Consistently, signal correlations increased with spatial receptive similarity (Fig. $8 E$, black curve). Therefore, the spatial receptive fields capture some of the local response diversity, albeit a small fraction of it (Fig. $8 E$, gray dots).

Finally, we studied how signal correlations are organized in cortical space. Correlations were strongest for nearby neurons and weakest for distant neurons (Fig. $8 F$ ). This relationship could reflect retinotopy (Figs. 6, 7), or it could reflect the clustering of other response properties, including those not represented by the spatial receptive fields. To distinguish between these possibilities, we calculated the average signal correlation as function of cortical distance and receptive-field similarity (Fig. 8G). For a given degree of similarity, the average correlation was approximately constant with cortical distance. Thus, the proximity of the neurons per se has little impact on signal correlations, and the fall off of correlations with cortical distance is explained by the layout of spatial receptive fields. This also indicates that the response diversity not captured by the receptive fields either is not clustered or is in register with the map of receptive fields over cortex.

\section{Discussion}

We have used calcium imaging to study the functional diversity and organization of local neural populations in mouse visual cortex. High-speed imaging and advanced analysis methods allowed the study of local population activity with a degree of detail approaching that of electrode recordings. Critical to these experiments were the technology to image wide fields at video rate (Fig. 1), the methods to estimate spike-related cellular calcium activity (Fig. 2) and the wavelet stimulus to characterize populations (Fig. 4). Together, these methods expand the range of experiments that can be conducted with two-photon calcium imaging, allowing for characterization of populations using harmonic analysis (Fig. 3), reverse correlation (Fig. 4), functional modeling (Figs. 5-7), and measures of correlated responses to the wavelet stimulus (Fig. 8).

\section{Functional diversity of local visual cortical populations}

We observed pronounced functional differences between the responses of excitatory and inhibitory populations in mouse visual cortex. When studied with grating stimuli, inhibitory neurons had less diverse responses than excitatory neurons (Figs. 3-6), showing a weak tuning for orientation and complex-like responses. These results are consistent with studies of the responses of fast-spiking cells (Niell and Stryker, 2008) and parvalbuminpositive neurons (Sohya et al., 2007; Kerlin et al., 2010; Liu et al., 2010; Ma et al., 2010; Zariwala et al., 2011) in mouse visual cortex but do not exclude the possibility that distinct subpopulations of GABAergic neurons may have more diverse properties (Ma et al., 2010; Runyan et al., 2010).

Together, these functional differences suggest that inhibitory neurons may inherit their visual properties by promiscuously summing signals from surrounding excitatory neurons (Kerlin et al., 2010; Bock et al., 2011; Fino and Yuste, 2011). Weak tuning of GABAergic responses in mouse visual cortex could be explained by summing the responses of a range of highly tuned cells. Likewise, complex-like properties could reflect the summing of responses of neurons with overlapping $\mathrm{ON}$ and $\mathrm{OFF}$ receptive-field subregions. In cat visual cortex, fast-spiking neurons have more diverse response properties. Some of these neurons have been shown to have weak tuning and complex-like receptive fields (Hirsch et al., 2003; Nowak et al., 2008), but orientation-tuned and simple cell responses have also been observed. This difference may reflect the difference in functional architecture between cats and rodents.

In contrast to inhibitory neurons, excitatory neurons in mouse visual cortex showed high diversity. The responses of pairs of neurons to the wavelet stimulus were on average only weakly correlated. Some but not all of this diversity could be attributed to the spatial receptive fields (Figs. 5-7). The remainder likely reflects differences in spatiotemporal summation (Fig. 5), additional nonlinear mechanisms (Vinje and Gallant, 2000; Haider et al., 2010), or noise in the measurements. This diversity is reminiscent of that observed in mouse barrel (Sato et al., 2007) and auditory cortices (Bandyopadhyay et al., 2010; Rothschild et al., 2010) where neurons with distinct properties are found next to each other.

The high diversity in local population responses has important implications for coding and decoding by downstream neurons. Theoretical studies suggest that receptive-field diversity may serve to establish a sparse and/or efficient visual code (Barlow, 1961; Simoncelli, 2003). The low correlations we observed are consistent with this hypothesis, though they are not a direct test of it. Studies of decoding also indicate that summing re- 
sponses of several neurons with similar receptive fields can compensate for response variability (Paradiso, 1988; Vogels and Orban, 1990; Zohary, 1992; Mazurek and Shadlen, 2002; Seriès et al., 2004). We have shown that very few such neurons are likely to exist, let alone project to the same target area. A plausible decoding scheme should therefore consider how to pool responses from diverse populations with little overlap in their tuning properties. Data like those presented here could yield more realistic models of visual processing and allow prediction of psychophysical performance in tasks involving complex visual stimuli.

\section{Local organization of visual cortical populations}

We have found little evidence for organization of visual cortical responses at small scales (i.e., the scale of a few tens of micrometers). Spatial receptive fields were locally disorganized (Fig. 6A), and responses of nearby neurons at times differed strongly (Fig. 8). While there was a weak tendency for ON and OFF subregions to fall over distinct regions of the visual field (Fig. 6B) (Smith and Häusser, 2010), receptive fields were otherwise arranged as if they sampled orientation and phase randomly (Fig. 6E).

Receptive fields of nearby cells were scattered over a region of visual space of about half a receptive-field size (Fig. 7). This scatter is about half that inferred by the hand-mapped receptive fields of consecutive units encountered in vertical electrode penetrations (Dräger, 1975) but is on par with the scatter of ON or OFF receptive-field subregions measured with calcium imaging (Smith and Häusser, 2010). This scatter is also consistent with the scatter observed in visual cortex of cats (Das and Gilbert, 1997; DeAngelis et al., 1999; Hetherington and Swindale, 1999) and monkeys (Hubel and Wiesel, 1974; Dow et al., 1981) (ranging from one-quarter to one-half a receptive-field size).

The random organization of orientation preferences remains an unexplained difference between rodents and carnivores or primates, which have well organized orientation maps (Ohki et al., 2005; Van Hooser et al., 2005; Ohki and Reid, 2007). By contrast, retinotopic organization is a common feature of all species studies, and local retinotopic scatter has been well described in cats (DeAngelis et al., 1999). Because local diversity is so prevalent, and cortical maps are so variable, it has been proposed that functional architecture might not be an essential feature of cortical organization (Horton and Adams, 2005).

Despite the random local arrangement, some organization was visible at the scale of $<100 \mu \mathrm{m}$. Receptive-field similarity and overlap decreased with cortical distance (Figs. 6, 7E). As distance over cortex increased, receptive-field azimuth and elevation shifted along two approximately perpendicular axes (Fig. $7 B, C$ ), consistent with measurements at coarser scales in the mouse (Dräger, 1975; Schuett et al., 2002; Kalatsky and Stryker, 2003). To our knowledge, these measurements are the first to unambiguously demonstrate the mapping of receptive-field position in a small patch of cortex.

In fact, our results suggest that retinotopy may represent the sole source of tangential organization in mouse visual cortex. Signal correlations fell with cortical distance (Fig. $8 F$ ) but were approximately constant across distance once the spatial receptive fields were taken into account (Fig. 8G). While our experiments cannot exclude organization of other stimulus features that are in register with the map of spatial receptive fields, it is likely that we would have been able to detect clustering at other scales. There was little indication for such clustering. Our results only hold for pairwise relationships between neurons. Higher-order patterns of activity have been observed locally in cortex (Ohiorhenuan et al., 2010). In the future, it would be interesting to study these patterns using calcium imaging.

\section{Random stimuli for population imaging}

Critical to our experiments was our ability to measure the receptive fields of dense populations of neurons (Fig. 4). Random stimuli are useful to quantify receptive fields (Jones and Palmer, 1987a; Reid and Shapley, 1992; Reid and Alonso, 1995; Reid et al., 1997; Ringach et al., 1997; DeAngelis et al., 1999). These stimuli, however, generally evoke weak responses (Liu et al., 2009; Ma et al., 2010), which may not be reliably detected with in vivo calcium imaging whether due to limitations in signal-to-noise ratio (Göbel and Helmchen, 2007) or to limitations of the calcium indicator (Kerr et al., 2005; Tian et al., 2009). Sparse random stimuli have been successfully applied to measure receptive fields from calcium signals (Smith and Häusser, 2010), but these stimuli can only drive a fraction of the neurons simultaneously. Structured dense stimuli such as natural scenes (Vinje and Gallant, 2000; David et al., 2004) or filtered Gaussian noise (Niell and Stryker, 2008) drive populations more effectively because they stimulate multiple receptive-field subregions from multiple neurons simultaneously. Rather than isotropic noise, our stimuli were made of wavelets, which, like visual cortical receptive fields, are oriented in space and slanted in space-time. This match contributes to the strong calcium responses we have observed (Fig. 4C) and to our ability to measure receptive fields for over half the labeled neurons (Fig. $6 \mathrm{~B}$ ). In contrast to uncorrelated noise, the wavelets are localized in space and in frequency so they can be tailored to stimulate populations with specific tuning properties.

\section{High-speed in vivo imaging methods}

Equally important was our ability to measure population activity in the face of brain motion and contamination from the surrounding neuropil (Figs. 1,2). For that purpose, the combination of high-speed raster scanning (Leybaert and Sanderson, 2001; Nguyen et al., 2001; Rochefort et al., 2009; Jia et al., 2010; Cheng et al., 2011), principal component analysis (Mitra and Pesaran, 1999; Mukamel et al., 2009), and nonlinear deconvolution (Vogelstein et al., 2010) was particularly powerful. Because each frame is scanned in $30 \mathrm{~ms}$ and because physiological motion from, for example, heartbeat or breathing is small and comparatively slow $(\leq 10 \mathrm{~Hz})$, motion in $x-y$ is approximately rigid and can be corrected with $2 \mathrm{D}$ registration. This alleviated the need for the more complex algorithms required at slow scan rates (Dombeck et al., 2007; Greenberg and Kerr, 2009). As suggested by Mukamel et al. (2009), principal component analysis was effective in correcting for the effects of $z$-motion (Fig. 2). This came without the complexities and the slow sampling rates of volumetric scanning (Kerlin et al., 2010). It allowed correction for brisk motion (e.g., heartbeat) and worked well if motion was not too large relative to the combined cell body size and optical point spread along the $z$-axis $(\sim 15 \mu \mathrm{m})$. In the future, these techniques may be used for imaging in awake animals where brain motion can be severe and often correlated with behavioral responses (Dombeck et al., 2007, 2010; Sawinski et al., 2009; Andermann et al., 2010).

By comparison, optimized line scans (Göbel et al., 2007; Valmianski et al., 2010) and random access scanning (Grewe and Helmchen, 2009) can achieve faster sampling rates but are more sensitive to brain motion, due to incomplete sampling of the imaged volume. These techniques also require sophisti- 
cated procedures, and/or user input, to label regions of interest and to generate scan trajectories (Valmianski et al., 2010), which is often difficult when the duration of the experiment is limited, or when imaging small, distributed cell processes (e.g., imaging dendrites and spines with genetically encoded calcium indicators) (Kuchibhotla et al., 2008; Tian et al., 2009; Jia et al., 2010).

Scanning a single plane at high speed allowed for reconstruction of firing rate responses (Figs. 2-4). Numerous algorithms for such inferences have been proposed (Yaksi and Friedrich, 2006; Greenberg et al., 2008; Vogelstein et al., 2009, 2010; Grewe et al., 2010; Smith and Häusser, 2010). We chose the method by Vogelstein et al. (2010) because it is extremely fast, yielding estimates of activity minutes after the data have been acquired. This enabled optimization of stimulus parameters during the experiment to obtain larger responses and better receptive-field estimates. The method is based on a generative model of calcium responses, which can be expanded to include the process that led to the responses, such as the linear filtering of the stimulus by receptive fields. In the future, we expect that similar approaches, which integrate both a model of neural responses and a model of somatic calcium fluorescence generation, will increase the power of calcium imaging experiments.

\section{References}

Alonso JM, Usrey WM, Reid RC (2001) Rules of connectivity between geniculate cells and simple cells in cat primary visual cortex. J Neurosci 21:4002-4015.

Andermann ML, Kerlin AM, Reid RC (2010) Chronic cellular imaging of mouse visual cortex during operant behavior and passive viewing. Front Cell Neurosci 4:3.

Bandyopadhyay S, Shamma SA, Kanold PO (2010) Dichotomy of functional organization in the mouse auditory cortex. Nat Neurosci $13: 361-368$

Barlow HB (1961) Possible principles underlying the transformation of sensory images. In: Sensory communication (Rosenblith WA, ed). Cambirdge, MA: MIT.

Bock DD, Lee WC, Kerlin AM, Andermann ML, Hood G, Wetzel AW, Yurgenson S, Soucy ER, Kim HS, Reid RC (2011) Network anatomy and in vivo physiology of visual cortical neurons. Nature 471:177-182.

Bonin V, Histed MH, Yurgenson S, Reid RC (2009) High-speed twophoton imaging of visually evoked activity in mouse visual cortex. Soc Neurosci Abstr 35:353.22.

Bonin V, Histed MH, Reid RC (2010) Receptive field mapping of local populations in mouse visual cortex using two-photon calcium imaging. Paper presented at Computational and Systems Neuroscience 2010, Salt Lake City, UT, February 25-March 2.

Bosking WH, Crowley JC, Fitzpatrick D (2002) Spatial coding of position and orientation in primary visual cortex. Nat Neurosci 5:874-882.

Cai D, DeAngelis GC, Freeman RD (1997) Spatiotemporal receptive field organization in the lateral geniculate nucleus of cats and kittens. J Neurophysiol 78:1045-1061.

Carandini M, Heeger DJ, Movshon JA (1997) Linearity and normalization in simple cells of the macaque primary visual cortex. J Neurosci 17:8621-8644.

Chen Y, Geisler WS, Seidemann E (2006) Optimal decoding of correlated neural population responses in the primate visual cortex. Nat Neurosci 9:1412-1420.

Cheng A, Gonçalves JT, Golshani P, Arisaka K, Portera-Cailliau C (2011) Simultaneous two-photon calcium imaging at different depths with spatiotemporal multiplexing. Nat Methods 8:139-142.

Das A, Gilbert CD (1997) Distortions of visuotopic map match orientation singularities in primary visual cortex. Nature 387:594-598.

David SV, Vinje WE, Gallant JL (2004) Natural stimulus statistics alter the receptive field structure of $\mathrm{vl}$ neurons. J Neurosci 24:6991-7006.

DeAngelis GC, Ghose GM, Ohzawa I, Freeman RD (1999) Functional micro-organization of primary visual cortex: receptive field analysis of nearby neurons. J Neurosci 19:4046-4064.
Di Lorenzo PM (1989) Across unit patterns in the neural response to taste: vector space analysis. J Neurophysiol 62:823-833.

Dombeck DA, Khabbaz AN, Collman F, Adelman TL, Tank DW (2007) Imaging large-scale neural activity with cellular resolution in awake, mobile mice. Neuron 56:43-57.

Dombeck DA, Harvey CD, Tian L, Looger LL, Tank DW (2010) Functional imaging of hippocampal place cells at cellular resolution during virtual navigation. Nat Neurosci 13:1433-1440.

Dow BM, Snyder AZ, Vautin RG, Bauer R (1981) Magnification factor and receptive field size in foveal striate cortex of the monkey. Exp Brain Res 44:213-228.

Dräger UC (1975) Receptive fields of single cells and topography in mouse visual cortex. J Comp Neurol 160:269-290.

Fino E, Yuste R (2011) Dense inhibitory connectivity in neocortex. Neuron 69:1188-1203.

Gao E, DeAngelis GC, Burkhalter A (2010) Parallel input channels to mouse primary visual cortex. J Neurosci 30:5912-5926.

Gawne TJ, Richmond BJ (1993) How independent are the messages carried by adjacent inferior temporal cortical neurons? J Neurosci 13:2758-2771.

Göbel W, Helmchen F (2007) In vivo calcium imaging of neural network function. Physiology (Bethesda) 22:358-365.

Göbel W, Kampa BM, Helmchen F (2007) Imaging cellular network dynamics in three dimensions using fast 3D laser scanning. Nat Methods 4:73-79.

Greenberg DS, Kerr JN (2009) Automated correction of fast motion artifacts for two-photon imaging of awake animals. J Neurosci Methods 176:1-15.

Greenberg DS, Houweling AR, Kerr JN (2008) Population imaging of ongoing neuronal activity in the visual cortex of awake rats. Nat Neurosci 11:749-751.

Grewe BF, Helmchen F (2009) Optical probing of neuronal ensemble activity. Curr Opin Neurobiol 19:520-529.

Grewe BF, Langer D, Kasper H, Kampa BM, Helmchen F (2010) High-speed in vivo calcium imaging reveals neuronal network activity with nearmillisecond precision. Nat Methods 7:399-405.

Grinvald A, Lieke EE, Frostig RD, Hildesheim R (1994) Cortical pointspread function and long-range lateral interactions revealed by real-time optical imaging of macaque monkey primary visual cortex. J Neurosci 14:2545-2568.

Guizar-Sicairos M, Thurman ST, Fienup JR (2008) Efficient subpixel image registration algorithms. Opt Lett 33:156-158.

Haider B, Krause MR, Duque A, Yu Y, Touryan J, Mazer JA, McCormick DA (2010) Synaptic and network mechanisms of sparse and reliable visual cortical activity during nonclassical receptive field stimulation. Neuron 65:107-121.

Halko N, Martinsson P-G, Tropp JA (2011) Finding structure with randomness: probabilistic algorithms for constructing approximate matrix decompositions. SIAM Rev Soc Ind Appl Math 53:217-288.

Hetherington PA, Swindale NV (1999) Receptive field and orientation scatter studied by tetrode recordings in cat area 17. Vis Neurosci 16:637-652.

Hirsch JA, Martinez LM, Pillai C, Alonso JM, Wang Q, Sommer FT (2003) Functionally distinct inhibitory neurons at the first stage of visual cortical processing. Nat Neurosci 6:1300-1308.

Horton JC, Adams DL (2005) The cortical column: a structure without a function. Philos Trans R Soc Lond B Biol Sci 360:837-862.

Hubel DH, Wiesel TN (1959) Receptive fields of single neurones in the cat's striate cortex. J Physiol 148:574-591.

Hubel DH, Wiesel TN (1962) Receptive fields, binocular interaction and functional architecture in the cat's visual cortex. J Physiol 160:106-154.

Hubel DH, Wiesel TN (1968) Receptive fields and functional architecture of monkey striate cortex. J Physiol (London) 195:215-243.

Hubel DH, Wiesel TN (1974) Uniformity of monkey striate cortex: a parallel relationship between field size, scatter, and magnification factor. J Comp Neurol 158:295-305.

Jia H, Rochefort NL, Chen X, Konnerth A (2010) Dendritic organization of sensory input to cortical neurons in vivo. Nature 464:1307-1312.

Jones JP, Palmer LA (1987a) The two-dimensional spatial structure of simple receptive fields in cat striate cortex. J Neurophysiol 58:1187-1211.

Jones JP, Palmer LA (1987b) An evaluation of the two-dimensional Gabor filter model of simple receptive fields in cat striate cortex. J Neurophysiol 58:1233-1258. 
Kalatsky VA, Stryker MP (2003) New paradigm for optical imaging: temporally encoded maps of intrinsic signal. Neuron 38:529-545.

Kerlin AM, Andermann ML, Berezovskii VK, Reid RC (2010) Broadly tuned response properties of diverse inhibitory neuron subtypes in mouse visual cortex. Neuron 67:858-871.

Kerr JN, Greenberg D, Helmchen F (2005) Imaging input and output of neocortical networks in vivo. Proc Natl Acad Sci U S A 102:14063-14068.

Ko H, Hofer SB, Pichler B, Buchanan KA, Sjöström PJ, Mrsic-Flogel TD (2011) Functional specificity of local synaptic connections in neocortical networks. Nature 473:87-91.

Kuchibhotla KV, Goldman ST, Lattarulo CR, Wu HY, Hyman BT, Bacskai BJ (2008) Abeta plaques lead to aberrant regulation of calcium homeostasis in vivo resulting in structural and functional disruption of neuronal networks. Neuron 59:214-225.

Leybaert L, Sanderson MJ (2001) Intercellular calcium signaling and flash photolysis of caged compounds. A sensitive method to evaluate gap junctional coupling. Methods Mol Biol 154:407-430.

Liu BH, Li P, Li YT, Sun YJ, Yanagawa Y, Obata K, Zhang LI, Tao HW (2009) Visual receptive field structure of cortical inhibitory neurons revealed by two-photon imaging guided recording. J Neurosci 29:10520-10532.

Liu BH, Li P, Sun YJ, Li YT, Zhang LI, Tao HW (2010) Intervening inhibition underlies simple-cell receptive field structure in visual cortex. Nat Neurosci 13:89-96.

Ma WP, Liu BH, Li YT, Huang ZJ, Zhang LI, Tao HW (2010) Visual representations by cortical somatostatin inhibitory neurons-selective but with weak and delayed responses. J Neurosci 30:14371-14379.

Mangini NJ, Pearlman AL (1980) Laminar distribution of receptive field properties in the primary visual cortex of the mouse. J Comp Neurol 193:203-222.

Mazurek ME, Shadlen MN (2002) Limits to the temporal fidelity of cortical spike rate signals. Nat Neurosci 5:463-471.

Mitra PP, Pesaran B (1999) Analysis of dynamic brain imaging data. Biophys J 76:691-708.

Movshon JA, Thompson ID, Tolhurst DJ (1978a) Spatial summation in the receptive fields of simple cells in the cat's striate cortex. J Physiol (London) 283:53-77.

Movshon JA, Thompson ID, Tolhurst DJ (1978b) Receptive field organization of complex cells in the cat's striate cortex. J Physiol (London) 283:79-99.

Mrsic-Flogel TD, Hofer SB, Ohki K, Reid RC, Bonhoeffer T, Hübener M (2007) Homeostatic regulation of eye-specific responses in visual cortex during ocular dominance plasticity. Neuron 54:961-972.

Mukamel EA, Nimmerjahn A, Schnitzer MJ (2009) Automated analysis of cellular signals from large-scale calcium imaging data. Neuron 63:747-760.

Nguyen QT, Callamaras N, Hsieh C, Parker I (2001) Construction of a twophoton microscope for video-rate $\mathrm{Ca}(2+)$ imaging. Cell Calcium 30:383-393.

Niell CM, Stryker MP (2008) Highly selective receptive fields in mouse visual cortex. J Neurosci 28:7520-7536.

Nimmerjahn A, Kirchhoff F, Kerr JN, Helmchen F (2004) Sulforhodamine 101 as a specific marker of astroglia in the neocortex in vivo. Nat Methods $1: 31-37$.

Nowak LG, Sanchez-Vives MV, McCormick DA (2008) Lack of orientation and direction selectivity in a subgroup of fast-spiking inhibitory interneurons: cellular and synaptic mechanisms and comparison with other electrophysiological cell types. Cereb Cortex 18:1058-1078.

Ohiorhenuan IE, Mechler F, Purpura KP, Schmid AM, Hu Q, Victor JD (2010) Sparse coding and high-order correlations in fine-scale cortical networks. Nature 466:617-621.

Ohki K, Reid RC (2007) Specificity and randomness in the visual cortex. Curr Opin Neurobiol 17:401-407.

Ohki K, Chung S, Ch'ng YH, Kara P, Reid RC (2005) Functional imaging with cellular resolution reveals precise micro-architecture in visual cortex. Nature 433:597-603.

Paradiso MA (1988) A theory for the use of visual orientation information which exploits the columnar structure of striate cortex. Biol Cybern 58:35-49.

Priebe NJ, Ferster D (2008) Inhibition, spike threshold, and stimulus selectivity in primary visual cortex. Neuron 57:482-497.

Reich DS, Mechler F, Victor JD (2001) Independent and redundant information in nearby cortical neurons. Science 294:2566-2568.
Reid RC, Alonso JM (1995) Specificity of monosynaptic connections from thalamus to visual cortex. Nature 378:281-284.

Reid RC, Shapley RM (1992) Spatial structure of cone inputs to receptive fields in primate lateral geniculate nucleus. Nature 356:716-718.

Reid RC, Victor JD, Shapley RM (1997) The use of m-sequences in the analysis of visual neurons: linear receptive field properties. Vis Neurosci 14:1015-1027.

Ringach DL (2002) Spatial structure and symmetry of simple-cell receptive fields in macaque primary visual cortex. J Neurophysiol 88:455-463.

Ringach DL, Hawken MJ, Shapley R (1997) Dynamics of orientation tuning in macaque primary visual cortex. Nature 387:281-284.

Ringach DL, Hawken MJ, Shapley R (2002) Receptive field structure of neurons in monkey primary visual cortex revealed by stimulation with natural image sequences. J Vis 2:12-24.

Rochefort NL, Garaschuk O, Milos RI, Narushima M, Marandi N, Pichler B, Kovalchuk Y, Konnerth A (2009) Sparsification of neuronal activity in the visual cortex at eye-opening. Proc Natl Acad Sci U S A 106:1504915054.

Rothschild G, Nelken I, Mizrahi A (2010) Functional organization and population dynamics in the mouse primary auditory cortex. Nat Neurosci 13:353-360.

Runyan CA, Schummers J, Van Wart A, Kuhlman SJ, Wilson NR, Huang ZJ, Sur M (2010) Response features of parvalbumin-expressing interneurons suggest precise roles for subtypes of inhibition in visual cortex. Neuron 67:847-857.

Sagdullaev BT, DeMarco PJ, McCall MA (2004) Improved contact lens electrode for corneal ERG recordings in mice. Doc Ophthalmol 108:181-184.

Sato TR, Gray NW, Mainen ZF, Svoboda K (2007) The functional microarchitecture of the mouse barrel cortex. PLoS Biol 5:e189.

Sawinski J, Wallace DJ, Greenberg DS, Grossmann S, Denk W, Kerr JN (2009) Visually evoked activity in cortical cells imaged in freely moving animals. Proc Natl Acad Sci U S A 106:19557-19562.

Schuett S, Bonhoeffer T, Hübener M (2002) Mapping retinotopic structure in mouse visual cortex with optical imaging. J Neurosci 22:6549-6559.

Selesnick IW, Baraniuk RG, Kingsbury NC (2005) The dual-tree complex wavelet transform. IEEE Signal Process Mag 22:123-151.

Seriès P, Latham PE, Pouget A (2004) Tuning curve sharpening for orientation selectivity: coding efficiency and the impact of correlations. Nat Neurosci 7:1129-1135.

Simoncelli EP (2003) Vision and the statistics of the visual environment. Curr Opin Neurobiol 13:144-149.

Smetters D, Majewska A, Yuste R (1999) Detecting action potentials in neuronal populations with calcium imaging. Methods 18:215-221.

Smith SL, Häusser M (2010) Parallel processing of visual space by neighboring neurons in mouse visual cortex. Nat Neurosci 13:1144-1149.

Sohya K, Kameyama K, Yanagawa Y, Obata K, Tsumoto T (2007) GABAergic neurons are less selective to stimulus orientation than excitatory neurons in layer II/III of visual cortex, as revealed by in vivo functional $\mathrm{Ca}^{2+}$ imaging in transgenic mice. J Neurosci 27:2145-2149.

Stosiek C, Garaschuk O, Holthoff K, Konnerth A (2003) In vivo two-photon calcium imaging of neuronal networks. Proc Natl Acad Sci U S A 100:7319-7324.

Tamamaki N, Yanagawa Y, Tomioka R, Miyazaki J, Obata K, Kaneko T (2003) Green fluorescent protein expression and colocalization with calretinin, parvalbumin, and somatostatin in the GAD67-GFP knock-in mouse. J Comp Neurol 467:60-79.

Theunissen FE, David SV, Singh NC, Hsu A, Vinje WE, Gallant JL (2001) Estimating spatio-temporal receptive fields of auditory and visual neurons from their responses to natural stimuli. Network 12:289-316.

Thévenaz P, Ruttimann UE, Unser M (1998) A pyramid approach to subpixel registration based on intensity. IEEE Trans Image Process 7:27-41.

Tian L, Hires SA, Mao T, Huber D, Chiappe ME, Chalasani SH, Petreanu L, Akerboom J, McKinney SA, Schreiter ER, Bargmann CI, Jayaraman V, Svoboda K, Looger LL (2009) Imaging neural activity in worms, flies and mice with improved GCaMP calcium indicators. Nat Methods 6:875-881.

Tusa RJ, Palmer LA, Rosenquist AC (1978) The retinotopic organization of area 17 (striate cortex) in the cat. J Comp Neurol 177:213-235.

Usrey WM, Reppas JB, Reid RC (1999) Specificity and strength of retinogeniculate connections. J Neurophysiol 82:3527-3540.

Valmianski I, Shih AY, Driscoll JD, Matthews DW, Freund Y, Kleinfeld D 
(2010) Automatic identification of fluorescently labeled brain cells for rapid functional imaging. J Neurophysiol 104:1803-1811.

Van den Bergh G, Zhang B, Arckens L, Chino YM (2010) Receptive-field properties of V1 and V2 neurons in mice and macaque monkeys. J Comp Neurol 518:2051-2070.

Van Essen DC, Newsome WT, Maunsell JH (1984) The visual field representation in striate cortex of the macaque monkey: asymmetries, anisotropies, and individual variability. Vision Res 24:429-448.

Van Hooser SD, Heimel JA, Chung S, Nelson SB, Toth LJ (2005) Orientation selectivity without orientation maps in visual cortex of a highly visual mammal. J Neurosci 25:19-28.

Vinje WE, Gallant JL (2000) Sparse coding and decorrelation in primary visual cortex during natural vision. Science 287:1273-1276.

Vogels R, Orban GA (1990) How well do response changes of striate neurons signal differences in orientation: a study in the discrimination monkey. J Neurosci 10:3543-3558.

Vogelstein JT, Watson BO, Packer AM, Yuste R, Jedynak B, Paninski L (2009) Spike inference from calcium imaging using sequential Monte Carlo methods. Biophys J 97:636-655.

Vogelstein JT, Packer AM, Machado TA, Sippy T, Babadi B, Yuste R, Paninski
L (2010) Fast non-negative deconvolution for spike train inference from population calcium imaging. J Neurophysiol 104:3691-3704.

Wagor E, Mangini NJ, Pearlman AL (1980) Retinotopic organization of striate and extrastriate visual cortex in the mouse. J Comp Neurol 193:187-202.

Wang KH, Majewska A, Schummers J, Farley B, Hu C, Sur M, Tonegawa S (2006) In vivo two-photon imaging reveals a role of arc in enhancing orientation specificity in visual cortex. Cell 126:389-402.

Yaksi E, Friedrich RW (2006) Reconstruction of firing rate changes across neuronal populations by temporally deconvolved $\mathrm{Ca} 2+$ imaging. Nat Methods 3:377-383.

Yen SC, Baker J, Gray CM (2007) Heterogeneity in the responses of adjacent neurons to natural stimuli in cat striate cortex. J Neurophysiol 97:1326-1341.

Zariwala HA, Madisen L, Ahrens KF, Bernard A, Lein ES, Jones AR, Zeng H (2011) Visual tuning properties of genetically identified layer $2 / 3$ neuronal types in the primary visual cortex of cre-transgenic mice. Front Syst Neurosci 4:162.

Zohary E (1992) Population coding of visual stimuli by cortical neurons tuned to more than one dimension. Biol Cybern 66:265-272. 